\title{
Asymmetries in heavy quark pair and dijet production at an EIC
}

\author{
Daniël Boer, ${ }^{a}$ Piet J. Mulders, ${ }^{b}$ Cristian Pisano ${ }^{c, d}$ and Jian Zhou ${ }^{e, b}$ \\ ${ }^{a}$ Van Swinderen Institute for Particle Physics and Gravity, University of Groningen, \\ Nijenborgh 4, 9747 AG Groningen, The Netherlands \\ ${ }^{b}$ Nikhef and Department of Physics and Astronomy, VU University Amsterdam, \\ De Boelelaan 1081, NL-1081 HV Amsterdam, The Netherlands \\ ${ }^{c}$ Dipartimento di Fisica, Università di Pavia, \\ via Bassi 6, I-27100 Pavia, Italy \\ ${ }^{d}$ INFN Sezione di Pavia, \\ via Bassi 6, I-27100 Pavia, Italy \\ e School of physics, \& Key Laboratory of Particle Physics and Particle Irradiation (MOE), \\ Shandong University, \\ Jinan, Shandong 250100, China \\ E-mail: d.boer@rug.nl, mulders@few.vu.nl, cristian.pisano@unipv.it, \\ jzhou@sdu.edu.cn
}

ABSTRACT: Asymmetries in heavy quark pair and dijet production in electron-proton collisions allow studies of gluon TMDs in close analogy to studies of quark TMDs in semiinclusive DIS. Here we present expressions for azimuthal asymmetries for both unpolarized and transversely polarized proton cases and consider the maximal asymmetries allowed. The latter are found to be rather sizeable, except in certain kinematic limits which are pointed out. In addition, we consider the small- $x$ limit and expectations from a McLerranVenugopalan model for unpolarized and linearly polarized gluons and from a perturbative, large transverse momentum calculation for the T-odd gluon TMDs. Comparison to related observables at RHIC and LHC is expected to provide valuable information about the process dependence of the gluon TMDs. In particular this will offer the possibility of a sign change test of the gluon Sivers TMD and two other T-odd gluon TMDs. This provides additional motivation for studies of azimuthal asymmetries in heavy quark pair and dijet production at a future Electron-Ion Collider.

KeYwords: Deep Inelastic Scattering (Phenomenology), QCD Phenomenology

ArXIV EPRINT: 1605.07934 


\section{Contents}

1 Introduction 1

2 Gluon TMD definitions and small- $x$ expressions 2

$\begin{array}{lll}3 & \text { Heavy quark pair production } & 7\end{array}$

4 Sign change test of the T-odd gluon TMDs 14

$\begin{array}{lll}5 & \text { Dijet production } & 15\end{array}$

$\begin{array}{llr}6 & \text { Conclusions } & 17\end{array}$

\section{Introduction}

Heavy quark pair and dijet production in electron-proton collisions offer direct probes of the gluons inside the proton. They allow to map out detailed information about the momentum distribution of the gluons, including the transverse momentum dependence [1,2]. This is of particular interest in the case of transversely polarized protons, which are known to exhibit large left-right asymmetries. Such asymmetries have not yet been studied in heavy quark pair or dijet production in electron-proton collisions, but form a prominent part of the physics case for an Electron-Ion Collider (EIC) proposed in the U.S. [3, 4]. Especially the study of the gluon Sivers effect is of interest here. The Sivers effect [5] refers to the transverse momentum distribution of unpolarized quarks and gluons inside a transversely polarized proton, where the transverse momentum forms a $\sin \phi$ distribution around the transverse spin direction.

For transversely polarized protons there is a strong analogy between quark and gluon distributions. For both there is the Sivers effect already mentioned, but there is also a parallel between the helicity flip distributions of transversely polarized quarks and of linearly polarized gluons inside transversely polarized protons, for which there are two each. Even though the latter distributions have different chirality and $\mathrm{T}$ behavior, their transverse momentum structures are such that they lead to the same azimuthal asymmetries in certain processes. This parallel we will discuss explicitly for the quark asymmetries in semi-inclusive Deep Inelastic Scattering (SIDIS) process $\left(e p \rightarrow e^{\prime} h X\right)$ and the gluon asymmetries in open heavy quark pair production $\left(e p \rightarrow e^{\prime} Q \bar{Q} X\right)$.

The transverse momentum dependent distribution functions (TMDs) of gluons inside transversely polarized protons are also of interest because of their process dependence and their associated small $x$ behavior. TMDs are inherently process dependent, expressed through their gauge link dependence. For the processes under consideration here, the gauge links are exclusively future pointing, and can be related directly to the gluon TMDs appearing at LHC in Higgs or heavy scalar quarkonium production (see section 4). This 
in principle allows to cross check the results obtained at LHC and EIC for unpolarized protons and perhaps for transversely polarized protons too in case a polarized fixed target experiment, called AFTER@LHC [6, 7], will be performed in the future at LHC. The gluon TMDs appearing in these processes correspond to the Weiszäcker-Williams (WW) distributions at small $x[8,9]$. Recently it was pointed out in ref. [10] that unlike the dipole gluon TMDs (which have one future and one past pointing gauge link), the WW gluon TMDs for a transversely polarized proton are suppressed with respect to the unpolarized gluon TMD by a factor of $x$. This implies that the transverse spin asymmetries in heavy quark pair and dijet production in electron-proton collisions will become suppressed in the small- $x$ limit. A test of this at EIC would be very interesting, as the EIC can probe both small and large $x$ regions. At RHIC heavy quarkonia in the forward region are not accessible, but other processes can be studied that in the small- $x$ limit probe the dipole distributions that are not suppressed with respect to the unpolarized gluon TMD towards small $x$. Such processes may also be studied very precisely at large $x$ at AFTER@LHC [1113]. For a further discussion we refer to ref. [14]. Obtaining a consistent picture among the various processes at small and large $x$ is important for testing our understanding of transverse spin effects and the TMD formalism in general.

In this paper we will restrict to the WW-type functions that appear in the heavy quark pair and dijet production at an EIC. The outline of the paper is as follows. In section 2 we provide the definition of gluon TMDs in terms of QCD operators and discuss some phenomenological models which are relevant in the small- $x$ region. Details of the calculations of the cross section for heavy quark pair production in DIS are discussed in section 3, together with the expressions for the azimuthal asymmetries and their upper bounds. In section 4 we comment on the process dependence of the gluon TMDs involved. Our results for dijet production are given in section 5. Summary and conclusions are presented in section 6 .

\section{Gluon TMD definitions and small- $x$ expressions}

Transverse momentum dependent distribution functions (TMDs) of gluons inside a proton with four-momentum $P$ and spin vector $S$ are defined through a matrix element of a correlator of the gluon field strengths $F^{\mu \nu}(0)$ and $F^{\nu \sigma}(\xi)$, evaluated at fixed light-front (LF) time $\xi^{+}=\xi \cdot n=0$, where $n$ is a lightlike vector conjugate to $P$. Explicitly, the correlator is given by [15]

$$
\left.\Gamma_{g}^{\mu \nu}\left(x, \boldsymbol{p}_{T}\right)=\frac{n_{\rho} n_{\sigma}}{(P \cdot n)^{2}} \int \frac{d(\xi \cdot P) d^{2} \xi_{T}}{(2 \pi)^{3}} e^{i p \cdot \xi}\left\langle P, S\left|\operatorname{Tr}\left[F^{\mu \rho}(0) U_{[0, \xi]} F^{\nu \sigma}(\xi) U_{[\xi, 0]}^{\prime}\right]\right| P, S\right\rangle\right\rfloor_{\mathrm{LF}},
$$

where the gluon momentum $p$ is decomposed as $p=x P+p_{T}+p^{-} n$, while $U_{[0, \xi]}$ and $U_{[0, \xi]}^{\prime}$ are process dependent gauge links that ensure gauge invariance. The longitudinal and transverse component of the proton spin are defined through the Sudakov decomposition

$$
S^{\mu}=\frac{S_{L}}{M_{p}}\left(P^{\mu}-\frac{M_{p}^{2}}{P \cdot n} n^{\mu}\right)+S_{T}^{\mu},
$$

with $S_{L}^{2}+\boldsymbol{S}_{T}^{2}=1$ and $M_{p}$ being the proton mass. For an unpolarized $(U)$ and transversely polarized $(T)$ proton, the following parametrizations for the correlator in terms of gluon 
TMDs will be employed:

$$
\begin{aligned}
\Gamma_{U}^{\mu \nu}\left(x, \boldsymbol{p}_{T}\right)= & \frac{x}{2}\left\{-g_{T}^{\mu \nu} f_{1}^{g}\left(x, \boldsymbol{p}_{T}^{2}\right)+\left(\frac{p_{T}^{\mu} p_{T}^{\nu}}{M_{p}^{2}}+g_{T}^{\mu \nu} \frac{\boldsymbol{p}_{T}^{2}}{2 M_{p}^{2}}\right) h_{1}^{\perp g}\left(x, \boldsymbol{p}_{T}^{2}\right)\right\}, \\
\Gamma_{T}^{\mu \nu}\left(x, \boldsymbol{p}_{T}\right)= & \frac{x}{2}\left\{g_{T}^{\mu \nu} \frac{\epsilon_{T}^{\rho \sigma} p_{T \rho} S_{T \sigma}}{M_{p}} f_{1 T}^{\perp g}\left(x, \boldsymbol{p}_{T}^{2}\right)+i \epsilon_{T}^{\mu \nu} \frac{p_{T} \cdot S_{T}}{M_{p}} g_{1 T}^{g}\left(x, \boldsymbol{p}_{T}^{2}\right)\right. \\
& \left.+\frac{p_{T \rho} \epsilon_{T}^{\rho\{\mu} p_{T}^{\nu\}}}{2 M_{p}^{2}} \frac{p_{T} \cdot S_{T}}{M_{p}} h_{1 T}^{\perp g}\left(x, \boldsymbol{p}_{T}^{2}\right)-\frac{p_{T \rho} \epsilon_{T}^{\rho\{\mu} S_{T}^{\nu\}}+S_{T \rho} \epsilon_{T}^{\rho\{\mu} p_{T}^{\nu\}}}{4 M_{p}} h_{1 T}^{g}\left(x, \boldsymbol{p}_{T}^{2}\right)\right\},
\end{aligned}
$$

where we have introduced the symmetric and antisymmetric transverse projectors $g_{T}^{\mu \nu}=$ $g^{\mu \nu}-P^{\mu} n^{\nu} / P \cdot n-n^{\mu} P^{\nu} / P \cdot n$ and $\epsilon_{T}^{\mu \nu}=\epsilon_{T}^{\alpha \beta \mu \nu} P_{\alpha} n_{\beta} / P \cdot n$, with $\epsilon_{T}^{12}=+1$, respectively.

The symmetric part of the correlator $\Gamma_{T}$, i.e. $\left(\Gamma_{T}^{\mu \nu}+\Gamma_{T}^{\nu \mu}\right) / 2$, is parametrized by three gluon TMDs that are all T-odd. The naming scheme used here for the gluon TMDs is based on the analogy with the quarks [16], but it should be emphasized that the quark and gluon TMDs with the same name are not necessarily linked by evolution and can have quite different properties under symmetry operations. The $h$ functions for quarks are chiral-odd and do not mix with the chiral-even $h$ functions of the gluons. Below we will make use of the combination

$$
h_{1}^{g} \equiv h_{1 T}^{g}+\frac{\boldsymbol{p}_{T}^{2}}{2 M_{p}^{2}} h_{1 T}^{\perp g},
$$

which, despite its name, is not related to the well-known transversity distribution $h_{1}^{q}$ for quarks that has no gluonic analogue. Although both $h_{1}^{q}$ and $h_{1}^{g}$ denote helicity flip distributions, the quark distribution is chiral-odd, T-even, and survives after transverse momentum integration, while the gluon distribution is chiral-even, T-odd, and vanishes upon integration over transverse momentum. Both distributions require another helicity flip somewhere else in the process, which will require consideration of different types of processes. But as we will show, the angular dependences in such distinct processes can be the same, which in our view justifies the naming scheme.

In order to estimate the size of the asymmetries in the small- $x$ limit, we discuss here some aspects of the small- $x$ behavior of the various gluon TMDs, starting with the linearly polarized gluon distribution. It has been shown that both the dipole and WW type linearly polarized gluon distributions can be studied in the small- $x$ formalism as they both have the same $\ln 1 / x$ enhancement in the small $x$-region as the unpolarized gluon distributions. It is thus possible to compute them using a saturation model. For example, in the McLerranVenugopalan (MV) model [17], the WW type linearly polarized gluon distribution is given by $[18,19]$

$$
x h_{1}^{\perp g}\left(x, \boldsymbol{p}_{T}^{2}\right)=\frac{S_{\perp}}{2 \pi^{3} \alpha_{s}} \frac{M_{p}^{2}}{2 \boldsymbol{p}_{T}^{2}} \frac{N_{c}^{2}-1}{N_{c}} \int_{0}^{\infty} d r_{\perp} \frac{J_{2}\left(\left|\boldsymbol{p}_{T}\right| r_{\perp}\right)}{r_{\perp} \ln \frac{1}{r_{\perp}^{2} \Lambda_{\mathrm{QCD}}^{2}}}\left(1-e^{-\frac{r_{\perp}^{2} Q_{s}^{2}}{4}}\right),
$$

where the saturation scale is defined as $Q_{s}^{2}=\alpha_{s} N_{c} \mu_{A} \ln \frac{1}{r_{\perp}^{2} \Lambda_{Q C D}^{2}}$ and $S_{\perp}$ is the transverse area of the nucleon/nucleus. In the MV model, there is a relation $\mu_{A} S_{\perp}=\alpha_{s} 2 \pi A$, where $A$ 


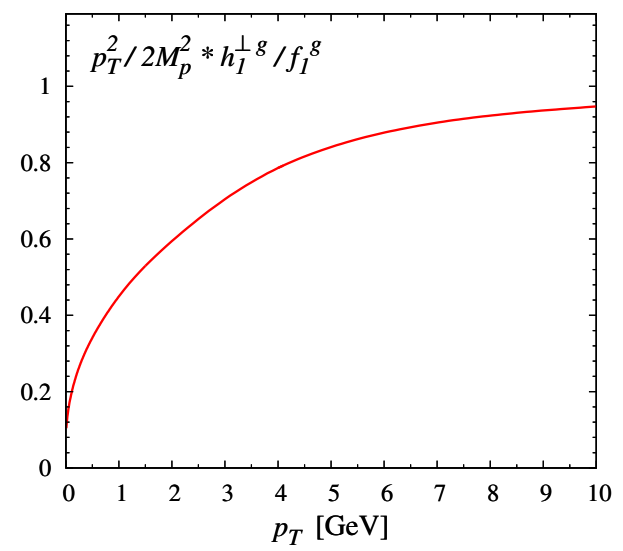

Figure 1. Ratio of the linearly polarized and unpolarized WW-type gluon TMDs in the MV model.

is the number of nucleons inside a nucleus, which is 1 in our case. Although the MV model is argued to work better for a large nucleus, its application to a nucleon target turns out to be quite successful phenomenologically $[20,21]$. For numerical estimations a regularized MV model expression is required for the small- $x$ WW type gluon TMDs [21-23],

$$
x h_{1}^{\perp g}\left(x, \boldsymbol{p}_{T}^{2}\right)=\frac{\left(N_{c}^{2}-1\right) \alpha_{s}}{\pi^{2}} \frac{M_{p}^{2}}{2 \boldsymbol{p}_{T}^{2}} \int_{0}^{\infty} d r_{\perp} \frac{J_{2}\left(\left|\boldsymbol{p}_{T}\right| r_{\perp}\right)}{r_{\perp} Q_{s 0}^{2} \ln \left[\frac{1}{r_{\perp}^{2} \Lambda_{\mathrm{QCD}}^{2}}+e\right]}\left\{1-e^{-\frac{r_{\perp}^{2} Q_{s 0}^{2}}{4} \ln \left[\frac{1}{r_{\perp}^{2} \Lambda_{\mathrm{QCD}}^{2}}+e\right]}\right\}
$$

where $Q_{s 0}^{2}$ is a starting saturation scale to be taken from fits to experimental data. The ratio between linearly polarized and unpolarized gluon distributions in the MV model is then given by

$$
\frac{\boldsymbol{p}_{T}^{2}}{2 M_{p}^{2}} \frac{h_{1}^{\perp g}\left(x, \boldsymbol{p}_{T}^{2}\right)}{f_{1}^{g}\left(x, \boldsymbol{p}_{T}^{2}\right)}=\frac{\int_{0}^{\infty} \frac{\mathrm{d} r_{\perp}}{r_{\perp}} J_{2}\left(\left|\boldsymbol{p}_{T}\right| r_{\perp}\right) \ln ^{-1}\left[\frac{1}{r_{\perp}^{2} \Lambda_{\mathrm{QCD}}^{2}}+e\right]\left\{1-e^{-\frac{r_{\perp}^{2} Q_{s 0}^{2}}{4} \ln \left[\frac{1}{r_{\perp}^{2} \Lambda_{\mathrm{QCD}}^{2}}+e\right]}\right\}}{\int_{0}^{\infty} \frac{\mathrm{d} r_{\perp}}{r_{\perp}} J_{0}\left(\left|\boldsymbol{p}_{T}\right| r_{\perp}\right)\left\{1-e^{-\frac{r_{\perp}^{2} Q_{s 0}^{2}}{4} \ln \left[\frac{1}{r_{\perp}^{2} \Lambda_{\mathrm{QCD}}^{2}}+e\right]}\right\}} .
$$

Numerically we find that $Q_{s 0}^{2}$ should be larger than about 1.1 times $C_{A} \Lambda_{\mathrm{QCD}}^{2} /\left(4 C_{F}\right)$, in order for the ratio to stay below 1 for all $p_{T}$ values (this is contrary to the claim in [19]). Alternatively one could consider replacing $e$ in the numerator by $e_{c} \cdot e$, and take $e_{c}$ to be sufficiently larger than 1 . In figure 1 we show the ratio for the case $Q_{s 0}^{2}=C_{A} / C_{F} \times$ $0.35 \mathrm{GeV}^{2}$ at $x=0.01$ and $\Lambda_{\mathrm{QCD}}=0.2 \mathrm{GeV}$. Here the value $0.35 \mathrm{GeV}^{2}$ is based on the GBW model fits to HERA data [24]. If one uses instead the value $0.104 \mathrm{GeV}^{2}$ in combination with $\Lambda_{\mathrm{QCD}}=0.241 \mathrm{GeV}$, as in [21], the resulting function would violate the positivity bound in a restricted region of intermediate transverse momentum. As can be seen from the figure, unlike the dipole case for which the ratio is saturated for all $p_{T}[18]$, the ratio in this WW 
case is not saturated for $p_{T}$ values below $10 \mathrm{GeV}$, but still sizeable except for very small $p_{T}$ values. Sizeable asymmetries may thus also be expected at EIC at small but not too small $x$.

Now we turn to discuss the behavior of the WW type T-odd gluon TMDs in the small $x$ limit. At large $p_{T}$, three T-odd WW type gluon TMDs inside a transversely polarized target can be perturbatively computed [10] following the standard collinear twist3 approach $[25,26]$. As mentioned in the introduction, these calculations have suggested that the WW gluon TMDs are suppressed by a factor of $x$ as compared to the corresponding dipole type ones. However, in ref. [10], only the asymptotic small- $x$ behavior of the WW gluon TMDs is discussed in the context of the collinear twist-3 approach without giving the full expressions. Below we present the complete results for these gluon distributions, which are relevant at moderately small $x$. One notices that such sub-asymptotic behavior can not be studied in the MV model, as in general, only the leading logarithm contribution is taken into account in saturation models. For the WW type gluon Sivers function, one has

$$
\begin{aligned}
f_{1 T}^{\perp g}\left(x, \boldsymbol{p}_{T}^{2}\right)= & \frac{C_{1} M_{p}}{p_{T}^{4}}\left\{\int_{x}^{1} \frac{\mathrm{d} z}{z} \sum_{q+\bar{q}}\left\{T_{F, q}(z, z) \frac{1+(1-\xi)^{2}}{\xi}-T_{F, q}(z, z-x) \frac{2-\xi}{\xi}\right\}\right. \\
& -16 \pi M_{p} \int_{x}^{1} \frac{d z}{z^{2}}\left\{\frac{\left(\xi^{2}-\xi+1\right)^{2}}{\xi(1-\xi)_{+}}[N(z, z)-N(z, 0)]+\frac{1+\xi^{2}}{2 \xi(1-\xi)_{+}} N(x, z)\right. \\
& \left.\left.-\frac{1+(1-\xi)^{2}}{2 \xi(1-\xi)_{+}} N(z, z-x)-\frac{\xi^{2}+(1-\xi)^{2}}{2 \xi(1-\xi)_{+}} N(x, x-z)\right\}\right\}
\end{aligned}
$$

where $\xi=x / z$ and $C_{1}=\frac{N_{c}}{2} \frac{\alpha_{s}}{2 \pi^{2}}$. Here $T_{F, q}(z, z)$ is the collinear twist-3 quark-gluon correlation function, commonly known as the Qiu-Sterman function [27], while $N\left(z_{1}, z_{2}\right)$ is a tri-gluon correlation function in which three gluons are in a symmetric color singlet state [28-30]. The tri-gluon correlation function has the symmetry properties: $N\left(x_{1}, x_{2}\right)=$ $N\left(x_{2}, x_{1}\right), N\left(x_{1}, x_{2}\right)=-N\left(-x_{1},-x_{2}\right)$, which below will be used to simplify the expressions. Terms proportional to $\delta(1-\xi)[N(z, z)-N(z, 0)]$ are ignored, as they are suppressed in the limit $x \rightarrow 0$. The large- $p_{T}$ expression of the gluon TMD $h_{1 T}^{g}\left(x, p_{T}^{2}\right)$ takes a similar form,

$$
\begin{aligned}
h_{1 T}^{g}\left(x, \boldsymbol{p}_{T}^{2}\right)= & C_{1} \frac{M_{p}}{p_{T}^{4}} 2\left\{\int_{x}^{1} \frac{\mathrm{d} z}{z} \sum_{q+\bar{q}}\left\{T_{F, q}(z, z) \frac{2-2 \xi}{\xi}-T_{F, q}(z, z-x) \frac{2-\xi}{\xi}\right\}\right. \\
& -16 \pi M_{p} \int_{x}^{1} \frac{d z}{z^{2}}\left\{\frac{1-\xi}{\xi} N(z, z)-\frac{\xi^{2}+(1-\xi)^{2}}{\xi(1-\xi)_{+}} N(z, 0)+\frac{1-\xi}{2 \xi} N(x, z)\right. \\
& \left.\left.-\frac{1+(1-\xi)^{2}}{2 \xi(1-\xi)_{+}} N(z, z-x)-\frac{1}{2 \xi(1-\xi)_{+}} N(x, x-z)\right\}\right\}
\end{aligned}
$$

where again the term proportional to $\delta(1-\xi)$ is ignored. Finally, the gluon TMD $h_{1 T}^{\perp g}\left(x, p_{T}^{2}\right)$ is given by

$$
h_{1 T}^{\perp g}\left(x, p_{T}^{2}\right)=C_{1} \frac{M_{p}}{p_{T}^{4}} \frac{2 M_{p}^{2}}{p_{T}^{2}} \int_{x}^{1} \frac{d z}{z}\left\{\sum_{q+\bar{q}} T_{F, q}(z, z)+T_{G}^{(+)}(z, z)\right\} \frac{4-4 \xi}{\xi},
$$

where the tri-gluon correlation function $T_{G}^{(+)}(z, z)$ is defined as $T_{G}^{(+)}(z, z) \equiv$ $-8 \pi M_{p}[N(z, z)-N(z, 0)] / z$. With these results, we can study the small- $x$ asymptotic behavior of the WW T-odd gluon TMDs. First of all, as observed in [10], there 
is a complete cancelation between the soft gluon pole contributions and the hard gluon pole contributions in the leading power of $1 / x$ for the gluon distributions $f_{1 T}^{\perp g}\left(x, p_{T}^{2}\right)$ and $h_{1 T}^{g}\left(x, p_{T}^{2}\right)$ in the small- $x$ limit. The leading contribution to the gluon TMD $h_{1 T}^{\perp g}\left(x, p_{T}^{2}\right)$ also vanishes by invoking the Burkardt sum rule [31, 32],

$$
\int_{0}^{1} d z\left\{\sum_{q+\bar{q}} T_{F, q}(z, z)+T_{G}^{(+)}(z, z)\right\}=0,
$$

which is stable under QCD evolution [33]. However, beyond the leading logarithm $\ln 1 / x$ approximation, there is no exact cancellation among the various contributions. In the small- $x$ limit, the subleading contributions to the gluon TMDs are given by

$$
\begin{aligned}
& f_{1 T}^{\perp g}\left(x, \boldsymbol{p}_{T}^{2}\right) \approx-\frac{C_{1} M_{p}}{p_{T}^{4}} \int_{x}^{1} \frac{d z}{z}\left\{\sum_{q+\bar{q}} T_{F, q}(z, z)+2 T_{G}^{(+)}(z, z)\right\}, \\
& h_{1 T}^{g}\left(x, \boldsymbol{p}_{T}^{2}\right) \approx-\frac{C_{1} M_{p}}{p_{T}^{4}} 2 \int_{x}^{1} \frac{d z}{z}\left\{\sum_{q+\bar{q}} T_{F, q}(z, z)+2 T_{G}^{(+)}(z, z)\right\}, \\
& h_{1 T}^{\perp g}\left(x, \boldsymbol{p}_{T}^{2}\right) \approx-\frac{C_{1} M_{p}}{p_{T}^{4}} 4 \frac{2 M_{p}^{2}}{p_{T}^{2}} \int_{x}^{1} \frac{d z}{z}\left\{\sum_{q+\bar{q}} T_{F, q}(z, z)+T_{G}^{(+)}(z, z)\right\} .
\end{aligned}
$$

We emphasize that the calculation presented here is based on the large- $p_{T}$ DGLAP-type formalism. Unlike the T-odd dipole type gluon TMDs [10, 34], these T-odd WW type gluon TMDs cannot be treated in the small- $x$ formalism due to the lack of the leading logarithmic contribution, at least not in a conventional way [35]. Nevertheless, we will use these compact results as guidance for modelling the WW gluon TMDs at small $x$ for all $p_{T}$. For example, if we assume the Burkardt sum rule were satisfied at each value of $z$, i.e. $\sum_{q+\bar{q}} T_{F, q}(z, z)+T_{G}^{(+)}(z, z)=0$, one obtains the simple result

$f_{1 T}^{\perp g}\left(x, \boldsymbol{p}_{T}^{2}\right)=\frac{1}{2} h_{1 T}^{g}\left(x, \boldsymbol{p}_{T}^{2}\right), \quad h_{1 T}^{\perp g}\left(x, \boldsymbol{p}_{T}^{2}\right)=0 \quad \Rightarrow h_{1}^{g}\left(x, \boldsymbol{p}_{T}^{2}\right)=2 f_{1 T}^{\perp g}\left(x, \boldsymbol{p}_{T}^{2}\right)$

If, on the other hand, we assume that the tri-gluon correlation were zero at some low initial scale and is purely dynamically generated by the Qiu-Sterman function $T_{F, q}$, one would have (note that $p_{T}^{2}=-\boldsymbol{p}_{T}^{2}$ )

$f_{1 T}^{\perp g}\left(x, \boldsymbol{p}_{T}^{2}\right)=\frac{1}{2} h_{1 T}^{g}\left(x, \boldsymbol{p}_{T}^{2}\right)=\frac{p_{T}^{2}}{8 M_{p}^{2}} h_{1 T}^{\perp g}\left(x, \boldsymbol{p}_{T}^{2}\right) \Rightarrow h_{1}^{g}\left(x, \boldsymbol{p}_{T}^{2}\right)=-2 f_{1 T}^{\perp g}\left(x, \boldsymbol{p}_{T}^{2}\right) \quad$ (Model II).

Finally, if for some reason it turns out that $\int_{x}^{1} d z T_{G}^{(+)}(z, z) / z \gg \sum_{q+\bar{q}} \int_{x}^{1} d z T_{F, q}(z, z) / z$, one obtains:

$$
f_{1 T}^{\perp g}\left(x, \boldsymbol{p}_{T}^{2}\right)=\frac{1}{2} h_{1 T}^{g}\left(x, \boldsymbol{p}_{T}^{2}\right)=\frac{p_{T}^{2}}{4 M_{p}^{2}} h_{1 T}^{\perp g}\left(x, \boldsymbol{p}_{T}^{2}\right) \quad \Rightarrow \quad h_{1}^{g}\left(x, \boldsymbol{p}_{T}^{2}\right)=0 \quad \text { (Model III) } .
$$

These models will be used below to obtain simple estimates for some ratio of asymmetries. All three models differ from the leading logarithmic result for the T-odd dipole functions $f_{1 T}^{\perp g}=\frac{1}{2} h_{1 T}^{g}=\frac{p_{T}^{2}}{2 M_{p}^{2}} h_{1 T}^{\perp g}=h_{1}^{g}$. 
The T-odd gluon TMDs have to satisfy the following positivity bounds [15]

$$
\begin{gathered}
\frac{\left|\boldsymbol{p}_{T}\right|}{M_{p}}\left|f_{1 T}^{\perp g}\left(x, \boldsymbol{p}_{T}^{2}\right)\right| \leq f_{1}^{g}\left(x, \boldsymbol{p}_{T}^{2}\right), \\
\frac{\left|\boldsymbol{p}_{T}\right|}{M_{p}}\left|h_{1}^{g}\left(x, \boldsymbol{p}_{T}^{2}\right)\right| \leq f_{1}^{g}\left(x, \boldsymbol{p}_{T}^{2}\right), \\
\frac{\left|\boldsymbol{p}_{T}\right|^{3}}{2 M_{p}^{3}}\left|h_{1 T}^{\perp g}\left(x, \boldsymbol{p}_{T}^{2}\right)\right| \leq f_{1}^{g}\left(x, \boldsymbol{p}_{T}^{2}\right) .
\end{gathered}
$$

In none of the three models we presented, one can satisfy the bounds for all three functions at the same time. This is in contrast to the dipole model for which all bounds can be saturated simultaneously.

It should be emphasized that for small $x$ the maximal values are not expected for the WW-type functions that are probed at EIC in heavy quark pair or dijet production. As mentioned, the WW T-odd gluon TMDs are suppressed by a factor of $x$ with respect to the WW unpolarized gluon TMD. The WW T-even gluon TMD $h_{1}^{\perp g}$ is not suppressed by a factor of $x$, but by a logarithm of $p_{T} / Q_{s}$ in the region $p_{T} \ll Q_{s}$ [18], whereas for $p_{T} \gg Q_{s}$ it was found to saturate the bound. This is compatible with the sizeable but not maximal effects we obtained in the MV model, figure 1 . This is why below we will show results for the MV model, rather than for $h_{1}^{\perp g}$ that saturates the positivity bound for all $p_{T}$.

\section{Heavy quark pair production}

The calculation of the cross section for the process

$$
e(\ell)+p(P, S) \rightarrow e\left(\ell^{\prime}\right)+Q\left(K_{1}\right)+\bar{Q}\left(K_{2}\right)+X,
$$

where the proton is polarized with polarization vector $S$ and the other particles are unpolarized, proceeds along the same lines of ref. [2], to which we refer for details. The heavy quark-antiquark pair in the final state is almost back-to-back in the plane perpendicular to the direction of the incoming proton and the photon exchanged in the reaction, that we take as $\hat{z}$-axis. Therefore one has $\left|q_{T}\right| \ll\left|K_{\perp}\right|$, where $q_{T} \equiv K_{1 \perp}+K_{2 \perp}$ and $K_{\perp} \equiv\left(K_{1 \perp}-K_{2 \perp}\right) / 2$, with $K_{i \perp}(i=1,2)$ being the transverse momenta of the heavy quark and antiquark, satisfying the relation $K_{i \perp}^{2}=-\boldsymbol{K}_{i \perp}^{2}$. This is often referred to as the back-to-back correlation limit. In a reference frame in which azimuthal angles are measured w.r.t. the lepton plane $\left(\phi_{\ell}=\phi_{\ell^{\prime}}=0\right)$, denoting by $\phi_{S}, \phi_{T}$ and $\phi_{\perp}$ the azimuthal angles of the three-vectors $\boldsymbol{S}_{T}$, $\boldsymbol{q}_{T}$ and $\boldsymbol{K}_{\perp}$, respectively, the cross section can be written as

$$
\frac{\mathrm{d} \sigma}{\mathrm{d} y_{1} \mathrm{~d} y_{2} \mathrm{~d} y \mathrm{~d} x_{B} \mathrm{~d}^{2} \boldsymbol{q}_{T} \mathrm{~d}^{2} \boldsymbol{K}_{\perp}} \equiv \mathrm{d} \sigma\left(\phi_{S}, \phi_{T}, \phi_{\perp}\right)=\mathrm{d} \sigma^{U}\left(\phi_{T}, \phi_{\perp}\right)+\mathrm{d} \sigma^{T}\left(\phi_{S}, \phi_{T}, \phi_{\perp}\right),
$$

where $y_{i}$ are the rapidities of the quarks, $y$ is the inelasticity variable, and $x_{B}=Q^{2} /(2 P \cdot q)$, with $Q^{2}=-q^{2}=-\left(\ell-\ell^{\prime}\right)^{2}$, is the Bjorken- $x$ variable. At leading order in perturbative QCD, we find

$$
\begin{aligned}
\mathrm{d} \sigma^{U}= & \mathcal{N}\left[A_{0}^{U}+A_{1}^{U} \cos \phi_{\perp}+A_{2}^{U} \cos 2 \phi_{\perp}+B_{0}^{U} \cos 2 \phi_{T}+B_{1}^{U} \cos \left(2 \phi_{T}-\phi_{\perp}\right)\right. \\
& \left.+B_{2}^{U} \cos 2\left(\phi_{T}-\phi_{\perp}\right)+B_{3}^{U} \cos \left(2 \phi_{T}-3 \phi_{\perp}\right)+B_{4}^{U} \cos \left(2 \phi_{T}-4 \phi_{\perp}\right)\right] \delta\left(1-z_{1}-z_{2}\right),
\end{aligned}
$$


and

$$
\begin{aligned}
\mathrm{d} \sigma^{T}= & \mathcal{N}\left|\boldsymbol{S}_{T}\right|\left\{\sin \left(\phi_{S}-\phi_{T}\right)\left[A_{0}^{T}+A_{1}^{T} \cos \phi_{\perp}+A_{2}^{T} \cos 2 \phi_{\perp}\right]+\cos \left(\phi_{S}-\phi_{T}\right)\left[B_{0}^{T} \sin 2 \phi_{T}\right.\right. \\
& \left.+B_{1}^{T} \sin \left(2 \phi_{T}-\phi_{\perp}\right)+B_{2}^{T} \sin 2\left(\phi_{T}-\phi_{\perp}\right)+B_{3}^{T} \sin \left(2 \phi_{T}-3 \phi_{\perp}\right)+B_{4}^{T} \sin \left(2 \phi_{T}-4 \phi_{\perp}\right)\right] \\
& +\left[B_{0}^{\prime T} \sin \left(\phi_{S}+\phi_{T}\right)+B_{1}^{\prime T} \sin \left(\phi_{S}+\phi_{T}-\phi_{\perp}\right)+B_{2}^{\prime T} \sin \left(\phi_{S}+\phi_{T}-2 \phi_{\perp}\right)\right. \\
& \left.\left.+B_{3}^{\prime T} \sin \left(\phi_{S}+\phi_{T}-3 \phi_{\perp}\right)+B_{4}^{\prime T} \sin \left(\phi_{S}+\phi_{T}-4 \phi_{\perp}\right)\right]\right\} \delta\left(1-z_{1}-z_{2}\right),
\end{aligned}
$$

where $z_{i}=P \cdot K_{i} / P \cdot q$, with $q \equiv \ell-\ell^{\prime}$. The normalization factor $\mathcal{N}$ is given by

$$
\mathcal{N}=\frac{\alpha^{2} \alpha_{s}}{\pi s M_{\perp}^{2}} \frac{1}{x_{B} y^{2}}
$$

with $s=(\ell+P)^{2}, M_{\perp}=\sqrt{M_{Q}^{2}+\boldsymbol{K}_{\perp}^{2}}$, where $M_{Q}$ is the (anti)quark mass. The explicit expressions of the terms $A_{l}^{U / T}$ in eqs. (3.3) and (3.4), with $l=0,1,2$, are given by

$$
A_{l}^{U}=e_{Q}^{2} T_{R} \mathcal{A}_{l}^{e g \rightarrow e Q \bar{Q}} f_{1}^{g}\left(x, \boldsymbol{q}_{T}^{2}\right), \quad A_{l}^{T}=A_{l}^{U} \frac{f_{1 T}^{\perp g}\left(x, \boldsymbol{q}_{T}^{2}\right)}{f_{1}^{g}\left(x, \boldsymbol{q}_{T}^{2}\right)},
$$

where $T_{R}=1 / 2$. We note that the three ratios $A_{l}^{T} / A_{l}^{U}$ are all equal to each other, i.e. to the ratio of the gluon Sivers function $f_{1 T}^{\perp g}$ and the unpolarized gluon distribution $f_{1}^{g}$. The hard scattering functions $\mathcal{A}_{l}^{e g \rightarrow e Q \bar{Q}}$ describe the interaction of an unpolarized gluon with a photon which can be in different polarization states [2],

$$
\begin{aligned}
& \mathcal{A}_{0}^{e g \rightarrow e Q \bar{Q}}=\left[1+(1-y)^{2}\right] \mathcal{A}_{U+L}^{\gamma^{*} g \rightarrow Q \bar{Q}}-y^{2} \mathcal{A}_{L}^{\gamma^{*} g \rightarrow Q \bar{Q}}, \\
& \mathcal{A}_{1}^{e g \rightarrow e Q \bar{Q}}=(2-y) \sqrt{1-y} \mathcal{A}_{I}^{\gamma^{*} g \rightarrow Q \bar{Q}}, \\
& \mathcal{A}_{2}^{e g \rightarrow e Q \bar{Q}}=2(1-y) \mathcal{A}_{T}^{\gamma^{*} g \rightarrow Q \bar{Q}},
\end{aligned}
$$

where the subscripts $U+L, L, I, T$ refer to the specific polarization of the photon $[2,36]$. Introducing the notation $z \equiv z_{1}, z_{2}=1-z, Q^{2}=-q^{2}$, we have

$$
\begin{aligned}
\mathcal{A}_{U+L}^{\gamma^{*} g \rightarrow Q \bar{Q}}= & \frac{1}{D^{3}}-\frac{z(1-z)}{D^{3}}\left\{2-4 \frac{M_{Q}^{2}}{M_{\perp}^{2}}+4 \frac{M_{Q}^{4}}{M_{\perp}^{4}}-\left[4 z(1-z)\left(2-3 \frac{M_{Q}^{2}}{M_{\perp}^{2}}\right)+2 \frac{M_{Q}^{2}}{M_{\perp}^{2}}\right] \frac{Q^{2}}{M_{\perp}^{2}}\right. \\
& \left.-z(1-z)[1-2 z(1-z)] \frac{Q^{4}}{M_{\perp}^{4}}\right\} \\
\mathcal{A}_{L}^{\gamma^{*} g \rightarrow Q \bar{Q}}= & 8 \frac{z^{2}(1-z)^{2}}{D^{3}}\left(1-\frac{M_{Q}^{2}}{M_{\perp}^{2}}\right) \frac{Q^{2}}{M_{\perp}^{2}}, \\
\mathcal{A}_{I}^{\gamma^{*} g \rightarrow Q \bar{Q}}= & 4 \sqrt{1-\frac{M_{Q}^{2}}{M_{\perp}^{2}}} \frac{z(1-z)(1-2 z)}{D^{3}} \frac{Q}{M_{\perp}}\left[1-z(1-z) \frac{Q^{2}}{M_{\perp}^{2}}-2 \frac{M_{Q}^{2}}{M_{\perp}^{2}}\right] \\
\mathcal{A}_{T}^{\gamma^{*} g \rightarrow Q \bar{Q}}= & 4 \frac{z(1-z)}{D^{3}}\left(1-\frac{M_{Q}^{2}}{M_{\perp}^{2}}\right)\left[z(1-z) \frac{Q^{2}}{M_{\perp}^{2}}+\frac{M_{Q}^{2}}{M_{\perp}^{2}}\right]
\end{aligned}
$$


with

$$
D \equiv D\left(z, \frac{Q^{2}}{M_{\perp}^{2}}\right)=1+z(1-z) \frac{Q^{2}}{M_{\perp}^{2}} .
$$

Analogously, the $B_{m}^{U}, B_{m}^{T}$ and $B_{m}^{\prime T}$, with $m=0,1,2,3,4$, describe the scattering of a linearly polarized gluon with a photon. They can be written as

$$
\begin{aligned}
B_{m}^{U} & =e_{Q}^{2} T_{R} \mathcal{B}_{m}^{e g \rightarrow e Q \bar{Q}} \frac{\boldsymbol{q}_{T}^{2}}{M_{p}^{2}} h_{1}^{\perp g}\left(x, \boldsymbol{q}_{T}^{2}\right), \quad B_{m}^{T}=B_{m}^{U} \frac{\left|\boldsymbol{q}_{T}\right|}{M_{p}} \frac{h_{1 T}^{\perp g}\left(x, \boldsymbol{q}_{T}^{2}\right)}{h_{1}^{\perp g}\left(x, \boldsymbol{q}_{T}^{2}\right)}, \\
B_{m}^{\prime T} & =B_{m}^{U} \frac{M_{p}}{\left|\boldsymbol{q}_{T}\right|} \frac{h_{1 T}^{g}\left(x, \boldsymbol{q}_{T}^{2}\right)}{h_{1}^{\perp g}\left(x, \boldsymbol{q}_{T}^{2}\right)},
\end{aligned}
$$

where

$$
\begin{aligned}
& \mathcal{B}_{2}^{e g \rightarrow e Q \bar{Q}}=\left[1+(1-y)^{2}\right] \mathcal{B}_{U+L}^{\gamma^{*} g \rightarrow Q \bar{Q}}-y^{2} \mathcal{B}_{L}^{\gamma^{*} g \rightarrow Q \bar{Q}}, \\
& \mathcal{B}_{j}^{e g \rightarrow e Q \bar{Q}}=(2-y) \sqrt{1-y} \mathcal{B}_{j I}^{\gamma^{*} g \rightarrow Q \bar{Q}} \quad(j=1,3), \\
& \mathcal{B}_{k}^{e g \rightarrow e Q \bar{Q}}=2(1-y) \mathcal{B}_{k T}^{\gamma^{*} g \rightarrow Q \bar{Q}} \quad(k=0,4),
\end{aligned}
$$

with

$$
\begin{aligned}
& \mathcal{B}_{U+L}^{\gamma^{*} g \rightarrow Q \bar{Q}}=\frac{z(1-z)}{D^{3}}\left\{[1-6 z(1-z)] \frac{Q^{2}}{M_{\perp}^{2}}-2 \frac{M_{Q}^{2}}{M_{\perp}^{2}}\right\}\left(1-\frac{M_{Q}^{2}}{M_{\perp}^{2}}\right), \\
& \mathcal{B}_{L}^{\gamma^{*} g \rightarrow Q \bar{Q}}=-4 \frac{z^{2}(1-z)^{2}}{D^{3}}\left(1-\frac{M_{Q}^{2}}{M_{\perp}^{2}}\right) \frac{Q^{2}}{M_{\perp}^{2}}, \\
& \mathcal{B}_{1 I}^{\gamma^{*} g \rightarrow Q \bar{Q}}=2 \frac{z(1-z)(1-2 z)}{D^{3}}\left[z(1-z) \frac{Q^{2}}{M_{\perp}^{2}}+\frac{M_{Q}^{2}}{M_{\perp}^{2}}\right] \sqrt{1-\frac{M_{Q}^{2}}{M_{\perp}^{2}} \frac{Q}{M_{\perp}}}, \\
& \mathcal{B}_{3 I}^{\gamma^{*} g \rightarrow Q \bar{Q}}=-2 \frac{z(1-z)(1-2 z)}{D^{3}}\left[1-\frac{M_{Q}^{2}}{M_{\perp}^{2}}\right]^{3 / 2} \frac{Q}{M_{\perp}}, \\
& \mathcal{B}_{0 T}^{\gamma^{*} g \rightarrow Q \bar{Q}}=-\frac{z(1-z)}{D^{3}}\left[z(1-z) \frac{Q^{2}}{M_{\perp}^{2}}+\frac{M_{Q}^{2}}{M_{\perp}^{2}}\right]^{2}, \\
& \mathcal{B}_{4 T}^{\gamma^{*} g \rightarrow Q \bar{Q}}=\frac{z(1-z)}{D^{3}}\left(1-\frac{M_{Q}^{2}}{M_{\perp}^{2}}\right)^{2} .
\end{aligned}
$$

The expressions involving $h_{1}^{\perp g}$ have appeared before in ref. [2], but here a few redefinitions are made in order to display the similarity between the contributions of the various $h$ functions. In particular, the following observable,

$$
\left|\left\langle\cos 2 \phi_{T}\right\rangle\right|=\left|\frac{\int \mathrm{d} \phi_{\perp} \mathrm{d} \phi_{T} \cos 2 \phi_{T} \mathrm{~d} \sigma}{\int \mathrm{d} \phi_{\perp} \mathrm{d} \phi_{T} \mathrm{~d} \sigma}\right|=\frac{\boldsymbol{q}_{T}^{2}\left|B_{0}^{U}\right|}{2 A_{0}^{U}}=\frac{\boldsymbol{q}_{T}^{2}}{2 M^{2}} \frac{\left|h_{1}^{\perp g}\left(x, \boldsymbol{p}_{T}^{2}\right)\right|}{f_{1}^{g}\left(x, \boldsymbol{p}_{T}^{2}\right)} \frac{\left|\mathcal{B}_{0}^{e g \rightarrow e Q \bar{Q}}\right|}{\mathcal{A}_{0}^{e g \rightarrow e Q \bar{Q}}}
$$

has been defined, which could provide direct access to $h_{1}^{\perp g}$. Using the earlier MV model expressions for the ratio between linearly polarized and unpolarized TMDs, in figure 2 we 

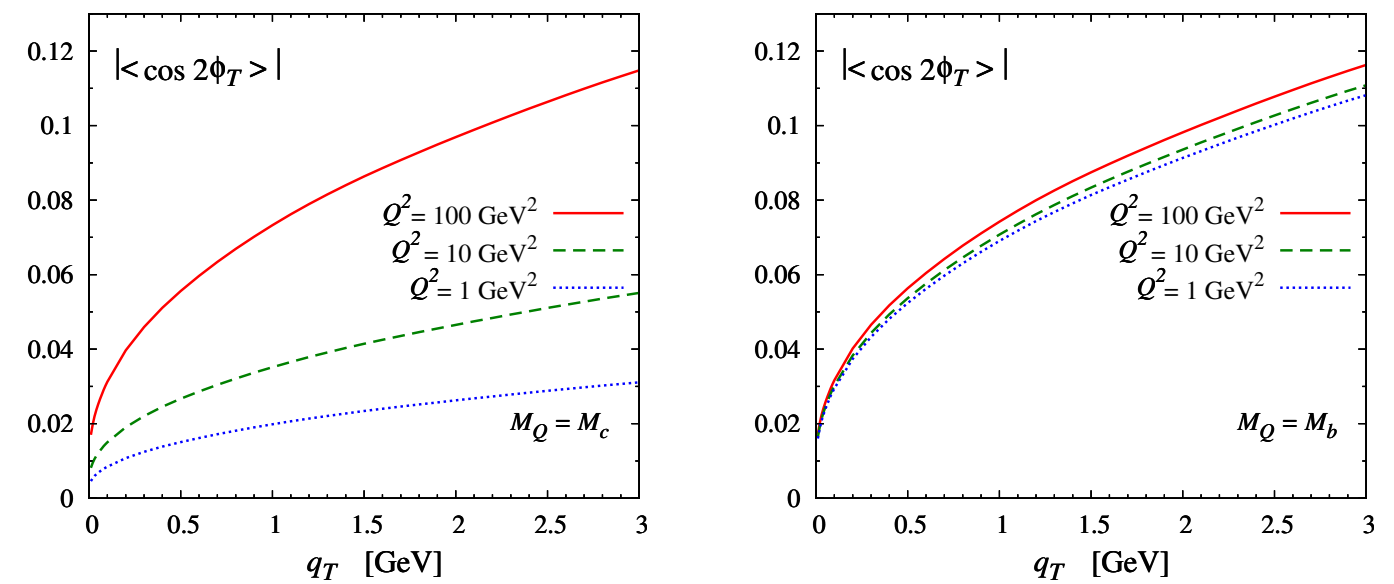

Figure 2. Estimates of the asymmetry $\left|\left\langle\cos 2 \phi_{T}\right\rangle\right|$ in the MV model, as a function of $q_{T} \equiv\left|\boldsymbol{q}_{T}\right|$, calculated at $\left|\boldsymbol{K}_{\perp}\right|=6 \mathrm{GeV}, z=0.5, y=0.1$ and at different values of $Q^{2}$, for charm (left panel) and bottom (right panel) production in the process $e p \rightarrow e^{\prime} Q \bar{Q} X$.

show our estimates for $\left|\left\langle\cos 2 \phi_{T}\right\rangle\right|$ at $z=0.5, y=0.1,\left|\boldsymbol{K}_{\perp}\right|=6 \mathrm{GeV}$ and $Q^{2} \geq 1 \mathrm{GeV}^{2}$, for both charm and bottom production. For this and the subsequent numerical studies we take the charm mass $M_{c}=1.3 \mathrm{GeV}$ and the bottom mass $M_{b}=4.2 \mathrm{GeV}$. We observe sizeable asymmetries in this MV model. Of course, the center of mass energy has to be sufficiently large for these curves to indeed be in the small- $x$ range. For an EIC the considered $\sqrt{s}$ ranges from about 20 to $150 \mathrm{GeV}$ [3], therefore $x_{B}=Q^{2} /(y s)<0.01$ for $y \geq 0.1$ requires $Q^{2}<\left(10^{-3}-10^{-2}\right) s$. For $\sqrt{s}=60 \mathrm{GeV}$ one should thus not consider the $Q^{2}=100 \mathrm{GeV}^{2}$ curve. We include the large $Q^{2}$ curves for a high energy EIC or for an analysis of HERA data which were taken at $\sqrt{s}=320 \mathrm{GeV}$. For larger $y$ and $\left|\boldsymbol{K}_{\perp}\right|$ values the asymmetries decrease. For $y=1\left|\left\langle\cos 2 \phi_{T}\right\rangle\right|$ vanishes identically.

Analogously, in figure 3 we provide our model estimates for the other asymmetry defined in ref. [2],

$\left|\left\langle\cos 2\left(\phi_{T}-\phi_{\perp}\right)\right\rangle\right|=\left|\frac{\int \mathrm{d} \phi_{\perp} \mathrm{d} \phi_{T} \cos 2\left(\phi_{T}-\phi_{\perp}\right) \mathrm{d} \sigma}{\int \mathrm{d} \phi_{\perp} \mathrm{d} \phi_{T} \mathrm{~d} \sigma}\right|=\frac{\boldsymbol{q}_{T}^{2}\left|B_{2}^{U}\right|}{2 A_{0}^{U}}=\frac{\boldsymbol{q}_{T}^{2}}{2 M^{2}} \frac{\left|h_{1}^{\perp g}\left(x, \boldsymbol{p}_{T}^{2}\right)\right|}{f_{1}^{g}\left(x, \boldsymbol{p}_{T}^{2}\right)} \frac{\left|\mathcal{B}_{2}^{e g \rightarrow e Q \bar{Q}}\right|}{\mathcal{A}_{0}^{e g \rightarrow e Q \bar{Q}}}$,

which is calculated at $z=0.5, y=0.3,\left|\boldsymbol{K}_{\perp}\right|=10 \mathrm{GeV}$ and $Q^{2} \geq 1 \mathrm{GeV}^{2}$. At large $q_{T}$ values the asymmetry is close to the maximal values allowed by the positivity bound. For larger $y$ and $\left|\boldsymbol{K}_{\perp}\right|$ values the asymmetries decrease again, but the asymmetry does not vanish at $y=1$.

If we introduce the combination $h_{1}^{g}$ of eq. (2.4), it turns out that the cross section in the transversely polarized proton case, when integrated over $\phi_{\perp}$, has only three independent azimuthal modulations: $\sin \left(\phi_{S}-\phi_{T}\right), \sin \left(\phi_{S}+\phi_{T}\right)$, and $\sin \left(\phi_{S}-3 \phi_{T}\right)$. Each one of them is related to a different T-odd gluon TMD. We observe that these angular structures and the TMDs they probe, are closely analogous to the case of quark asymmetries in SIDIS $\left(e p^{\uparrow} \rightarrow e^{\prime} h X\right)$, where the role of $\phi_{T}$ is played by $\phi_{h}$, cf. [37]. The same applies to the 

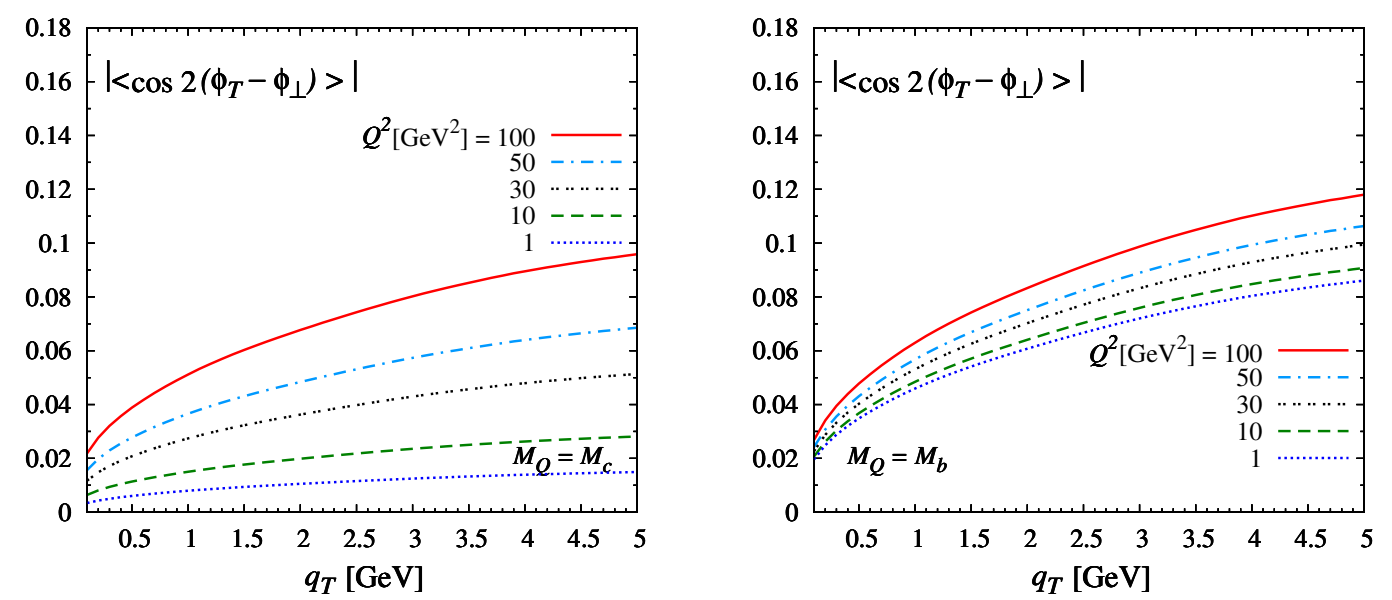

Figure 3. Estimates of the asymmetry $\left|\left\langle\cos 2\left(\phi_{T}-\phi_{\perp}\right)\right\rangle\right|$ in the MV model, as a function of $q_{T} \equiv\left|\boldsymbol{q}_{T}\right|$, calculated at $\left|\boldsymbol{K}_{\perp}\right|=10 \mathrm{GeV}, z=0.5, y=0.3$, and different values of $Q^{2}$, for charm (left panel) and bottom (right panel) production in the process $e p \rightarrow e^{\prime} Q \bar{Q} X$.

$\cos 2\left(\phi_{T}-\phi_{\perp}\right)$ asymmetry considered before.

In order to single out separate $\phi_{S}$ dependent terms, we define the following azimuthal moments

$$
\begin{aligned}
A_{N}^{W\left(\phi_{S}, \phi_{T}\right)} & \equiv 2 \frac{\int \mathrm{d} \phi_{T} \mathrm{~d} \phi_{\perp} W\left(\phi_{S}, \phi_{T}\right) \mathrm{d} \sigma_{T}\left(\phi_{S}, \phi_{T}, \phi_{\perp}\right)}{\int \mathrm{d} \phi_{T} \mathrm{~d} \phi_{\perp} \mathrm{d} \sigma_{U}\left(\phi_{T}, \phi_{\perp}\right)} \\
& =2 \frac{\int \mathrm{d} \phi_{T} \mathrm{~d} \phi_{\perp} W\left(\phi_{S}, \phi_{T}\right)\left[\mathrm{d} \sigma\left(\phi_{S}, \phi_{T}, \phi_{\perp}\right)-\mathrm{d} \sigma\left(\phi_{S}+\pi, \phi_{T}, \phi_{\perp}\right)\right]}{\int \mathrm{d} \phi_{T} \mathrm{~d} \phi_{\perp}\left[\mathrm{d} \sigma\left(\phi_{S}, \phi_{T}, \phi_{\perp}\right)+\mathrm{d} \sigma\left(\phi_{S}+\pi, \phi_{T}, \phi_{\perp}\right)\right]},
\end{aligned}
$$

where the denominator is given by

$$
\int \mathrm{d} \phi_{T} \mathrm{~d} \phi_{\perp} \mathrm{d} \sigma_{U}\left(\phi_{T}, \phi_{\perp}\right) \equiv \int \mathrm{d} \phi_{T} \mathrm{~d} \phi_{\perp} \frac{\mathrm{d} \sigma_{U}\left(\phi_{T}, \phi_{\perp}\right)}{\mathrm{d} y_{1} \mathrm{~d} y_{2} \mathrm{~d} y \mathrm{~d} x_{B} \mathrm{~d}^{2} \boldsymbol{q}_{T} \mathrm{~d}^{2} \boldsymbol{K}_{\perp}}=4 \pi \frac{\alpha^{2} \alpha_{s}}{s M_{\perp}^{2}} \frac{1}{x_{B} y^{2}} A_{0}^{U},
$$

and, since $A_{0}^{U}=A_{0}^{T} f_{1}^{g} / f_{1 T}^{\perp g}$, we obtain

$$
\begin{aligned}
A_{N}^{\sin \left(\phi_{S}-\phi_{T}\right)} & =\frac{\left|\boldsymbol{q}_{T}\right|}{M_{p}} \frac{A_{0}^{T}}{A_{0}^{U}}=\frac{\left|\boldsymbol{q}_{T}\right|}{M_{p}} \frac{f_{1 T}^{\perp g}\left(x, \boldsymbol{q}_{T}^{2}\right)}{f_{1}^{g}\left(x, \boldsymbol{q}_{T}^{2}\right)}, \\
A_{N}^{\sin \left(\phi_{S}+\phi_{T}\right)} & =\frac{\left|\boldsymbol{q}_{T}\right|}{M_{p}} \frac{B_{0}^{\prime T}}{A_{0}^{U}}=\frac{2(1-y) \mathcal{B}_{0 T}^{\gamma^{*} g \rightarrow Q \bar{Q}}}{\left[1+(1-y)^{2}\right] \mathcal{A}_{U+L}^{\gamma^{*} g \rightarrow Q \bar{Q}}-y^{2} \mathcal{A}_{L}^{\gamma^{*} g \rightarrow Q \bar{Q}}} \frac{\left|\boldsymbol{q}_{T}\right|}{M_{p}} \frac{h_{1}^{g}\left(x, \boldsymbol{q}_{T}^{2}\right)}{f_{1}^{g}\left(x, \boldsymbol{q}_{T}^{2}\right)}, \\
A_{N}^{\sin \left(\phi_{S}-3 \phi_{T}\right)} & =-\frac{\left|\boldsymbol{q}_{T}\right|^{3}}{M_{p}^{3}} \frac{B_{0}^{T}}{2 A_{0}^{U}}=-\frac{2(1-y) \mathcal{B}_{0 T}^{\gamma^{*} g \rightarrow Q \bar{Q}}}{\left[1+(1-y)^{2}\right] \mathcal{A}_{U+L}^{\gamma^{*} g \rightarrow Q \bar{Q}}-y^{2} \mathcal{A}_{L}^{\gamma^{*} g \rightarrow Q \bar{Q}}} \frac{\left|\boldsymbol{q}_{T}\right|^{3}}{2 M_{p}^{3}} \frac{h_{1 T}^{\perp g}\left(x, \boldsymbol{q}_{T}^{2}\right)}{f_{1}^{g}\left(x, \boldsymbol{q}_{T}^{2}\right)} .
\end{aligned}
$$

Note that the asymmetries in eqs. (3.20) and (3.21) vanish in the limit $y \rightarrow 1$ when the virtual photon is longitudinally polarized, which for $s / Q^{2} \rightarrow \infty$ corresponds to the limit 

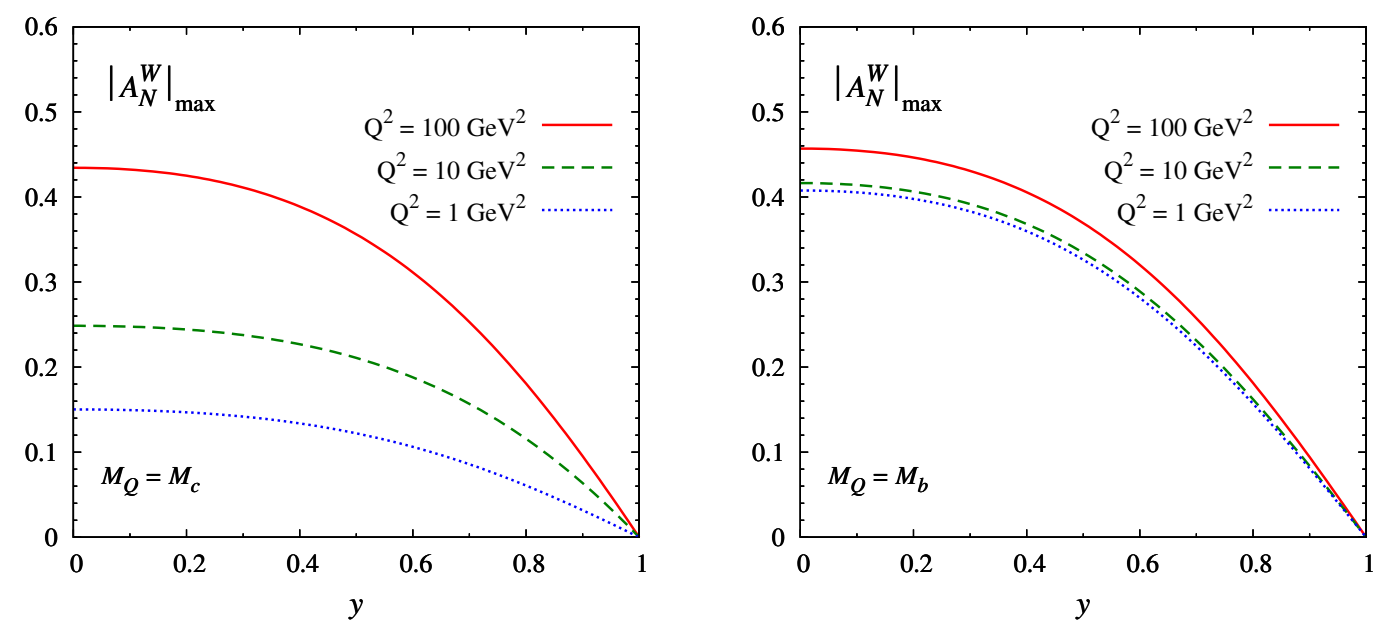

Figure 4. Upper bounds $\left|A_{N}^{W}\right|_{\max }$ on the asymmetries $A_{N}^{\sin \left(\phi_{S}+\phi_{T}\right)}$ and $A_{N}^{\sin \left(\phi_{S}-3 \phi_{T}\right)}$ for charm (left panel) and bottom (right panel) production in process $e p \rightarrow e^{\prime} Q \bar{Q} X$. Estimates are given as a function of $y$, at different values of $K_{\perp}>1 \mathrm{GeV}$ (chosen in such a way that the asymmetries are maximal) and $Q^{2}$, with $z=0.5$.

$x \rightarrow 0$. Based on the absence of the leading logarithmic term for the WW T-odd gluon TMDs, it is expected that also eq. (3.19) vanishes in this limit, but it does not do so automatically through a kinematic suppression term as for the other two asymmetries.

We note that a measurement of the ratio

$$
\frac{A_{N}^{\sin \left(\phi_{S}-3 \phi_{T}\right)}}{A_{N}^{\sin \left(\phi_{S}+\phi_{T}\right)}}=-\frac{\boldsymbol{q}_{T}^{2}}{2 M_{p}^{2}} \frac{h_{1 T}^{\perp g}\left(x, \boldsymbol{q}_{T}^{2}\right)}{h_{1}^{g}\left(x, \boldsymbol{q}_{T}^{2}\right)}
$$

would probe directly the relative magnitude of $h_{1 T}^{\perp g}$ and $h_{1}^{g}$ without dependences on any of the other kinematic variables in the process. In the small- $x$ models discussed in the previous section, one would obtain for this ratio: 0 in model I, -2 in model II, and $\infty$ in model III. These cases are all distinguishable from the dipole case, which would give 1. Note that this ratio need not be bounded between -1 and +1 .

As mentioned before, the WW T-odd gluon TMDs are not expected to satisfy the positivity bounds, but the latter can be used to determine the maximum values of the above defined asymmetries, and thereby exclude less promising kinematic regions. It can be easily seen that the Sivers asymmetry is bound to 1, while the asymmetries in eqs. (3.20) and (3.21) have the same upper bound, which we denote by $A_{N}^{W}$. The maximal values of $\left|A_{N}^{W}\right|$ for the latter are shown in figure 4 for charm and bottom production as a function of $y$, at $z=1 / 2, Q^{2}=1,10$ and $100 \mathrm{GeV}^{2}$. These maximal values are attained at some specific values of $\left|\boldsymbol{K}_{\perp}\right|$, which in this case corresponds to the lowest $\left|\boldsymbol{K}_{\perp}\right|$ value considered. The charm and bottom production upper bounds on $\left|A_{N}^{W}\right|$ as a function $\left|\boldsymbol{K}_{\perp}\right|$ are shown in figure 5, again at $z=1 / 2, y=0.1$ and $Q^{2}=1,10$ and $100 \mathrm{GeV}^{2}$. The upper bounds of these asymmetries are equal to the $R^{\prime}$ bounds of the weighted cross section $\left\langle\cos 2 \phi_{T}\right\rangle$, which are presented in figure 2 of ref. [2] for $y=0.01$. 

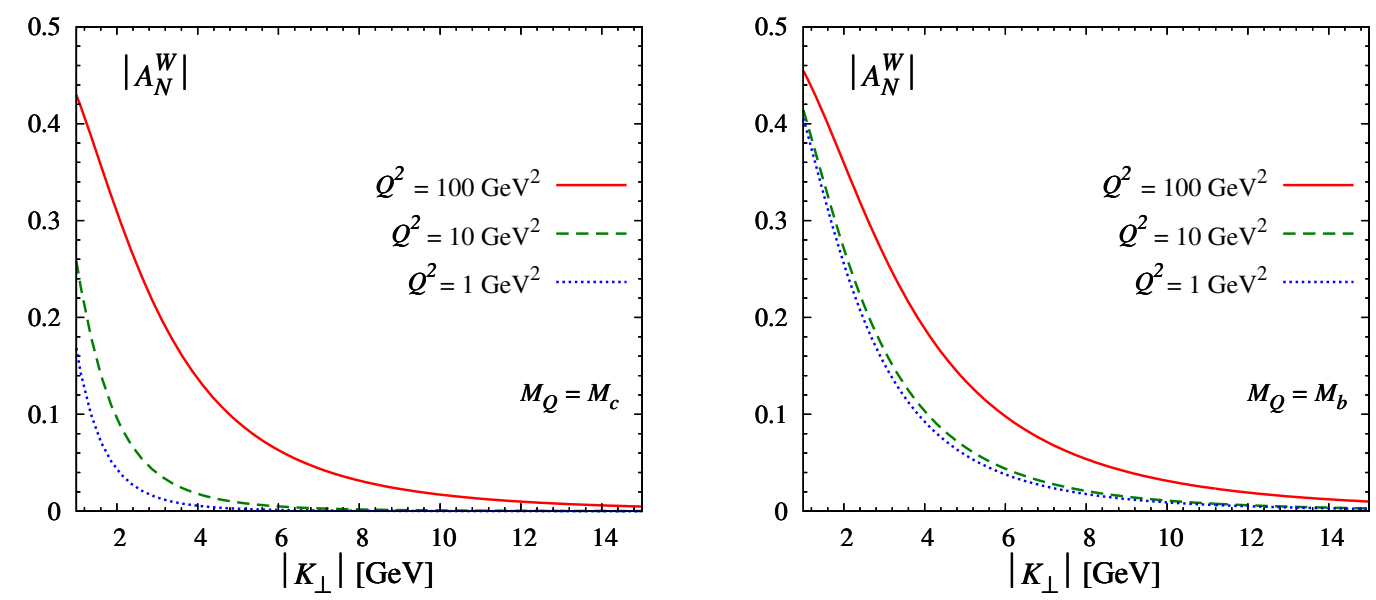

Figure 5. Estimates of $\left|A_{N}^{W}\right|$, with $W=\sin \left(\phi_{S}+\phi_{T}\right), \sin \left(\phi_{S}-3 \phi_{T}\right)$, for charm (left panel) and bottom (right panel) production in the process $e p \rightarrow e^{\prime} Q \bar{Q} X$, calculated at $z=0.5, y=0.1$ and different values of $Q^{2}$, as a function of $\left|\boldsymbol{K}_{\perp}\right|(>1 \mathrm{GeV})$.

Alternatively, one can define the azimuthal angles w.r.t. $\phi_{\perp}$ instead of $\phi_{\ell}$, which can then be integrated over. In this case, only the terms $A_{0}^{T}, B_{2}^{T}$ and $B_{2}^{\prime T}$ will contribute to the cross section in eq. (3.4). After introducing again the combination in eq. (2.4), one finds, in analogy to eqs. (3.19)-(3.21),

$$
\begin{aligned}
& A_{N}^{\sin \left(\phi_{S}^{\perp}-\phi_{T}^{\perp}\right)}=A_{N}^{\sin \left(\phi_{S}-\phi_{T}\right)}=\frac{\left|\boldsymbol{q}_{T}\right|}{M_{p}} \frac{A_{0}^{T}}{A_{0}^{U}}=\frac{\left|\boldsymbol{q}_{T}\right|}{M_{p}} \frac{f_{1 T}^{\perp g}\left(x, \boldsymbol{q}_{T}^{2}\right)}{f_{1}^{g}\left(x, \boldsymbol{q}_{T}^{2}\right)}, \\
& A_{N}^{\sin \left(\phi_{S}^{\perp}+\phi_{T}^{\perp}\right)}=\frac{\left|\boldsymbol{q}_{T}\right|}{M_{p}} \frac{B_{2}^{\prime T}}{A_{0}^{U}}=\frac{\left[1+(1-y)^{2}\right] \mathcal{B}_{U+L}^{\gamma^{*} g \rightarrow Q \bar{Q}}-y^{2} \mathcal{B}_{L}^{\gamma^{*} g \rightarrow Q \bar{Q}}}{\left[1+(1-y)^{2}\right] \mathcal{A}_{U+L}^{\gamma^{*} g \rightarrow Q \bar{Q}}-y^{2} \mathcal{A}_{L}^{\gamma^{*} g \rightarrow Q \bar{Q}}} \frac{\left|\boldsymbol{q}_{T}\right|}{M_{p}} \frac{h_{1}^{g}\left(x, \boldsymbol{q}_{T}^{2}\right)}{f_{1}^{g}\left(x, \boldsymbol{q}_{T}^{2}\right)}, \\
& A_{N}^{\sin \left(\phi_{S}^{\perp}-3 \phi_{T}^{\perp}\right)}=\frac{\left|\boldsymbol{q}_{T}\right|^{3}}{M_{p}^{3}} \frac{B_{2}^{T}}{2 A_{0}^{U}}=\frac{\left[1+(1-y)^{2}\right] \mathcal{B}_{U+L}^{\gamma^{*} g \rightarrow Q Q}-y^{2} \mathcal{B}_{L}^{\gamma^{*} g \rightarrow Q \bar{Q}}}{\left[1+(1-y)^{2}\right] \mathcal{A}_{U+L}^{\gamma^{*} g \rightarrow Q \bar{Q}}-y^{2} \mathcal{A}_{L}^{\gamma^{*} g \rightarrow Q \bar{Q}}} \frac{\left|\boldsymbol{q}_{T}\right|^{3}}{2 M_{p}^{3}} \frac{h_{1 T}^{\perp g}\left(x, \boldsymbol{q}_{T}^{2}\right)}{f_{1}^{g}\left(x, \boldsymbol{q}_{T}^{2}\right)} .
\end{aligned}
$$

As before, the asymmetries in eqs. (3.24)-(3.25) have the same upper bound $A_{N}^{W_{\perp}}$, presented in figures 6 and 7 for different values of the kinematic variables $\left|\boldsymbol{K}_{\perp}\right|, y, z, Q^{2}$. This bound is equal to $R$, the upper bound of $\left\langle\cos \left(2 \phi_{T}-\phi_{\perp}\right)\right\rangle$ discussed in figure 1 of ref. [2] for $y=0.01$. From figure 6 one sees that in this case the asymmetries do not need to vanish when $y \rightarrow 1$. This means that for small- $x$ studies the asymmetries $A_{N}^{W_{\perp}}$ may be of more interest than $A_{N}^{W}$. Due to the zero crossing that occurs in the maximal value of $\left|A_{N}^{W_{\perp}}\right|$, for charm production the asymmetry in the region of $Q^{2}$ between 5 and $10 \mathrm{GeV}^{2}$ is expected to be small. For bottom production this would apply to $Q^{2}$ closer to $100 \mathrm{GeV}^{2}$. Although these conclusions are based on the maximal asymmetries and therefore robust, the exact location of the zero crossing in $y$ may be affected by higher order corrections and thus may be a different function of $Q^{2}$ than obtained here. 

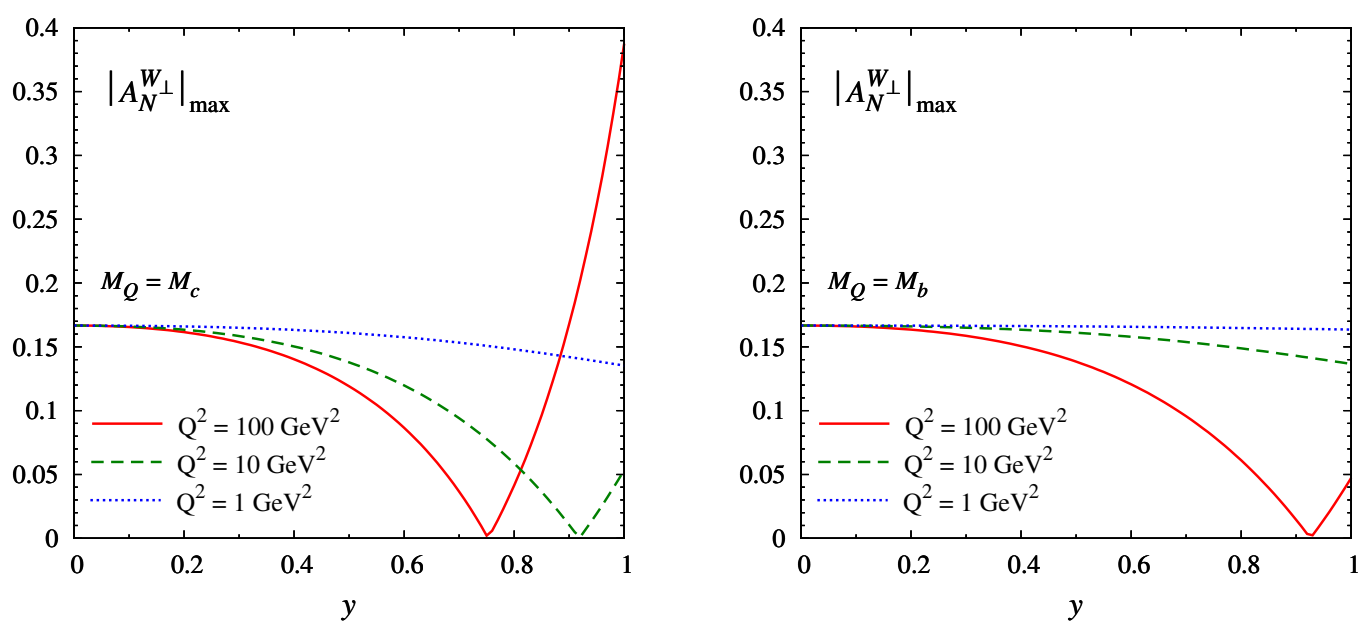

Figure 6. Same as in figure 4 , but for the upper bounds $\left|A_{N}^{W}\right|_{\max }$ of the asymmetries $A_{N}^{\sin \left(\phi_{S}^{\perp}+\phi_{T}^{\perp}\right)}$ and $A_{N}^{\sin \left(\phi_{S}^{\perp}-3 \phi_{T}^{\perp}\right)}$.
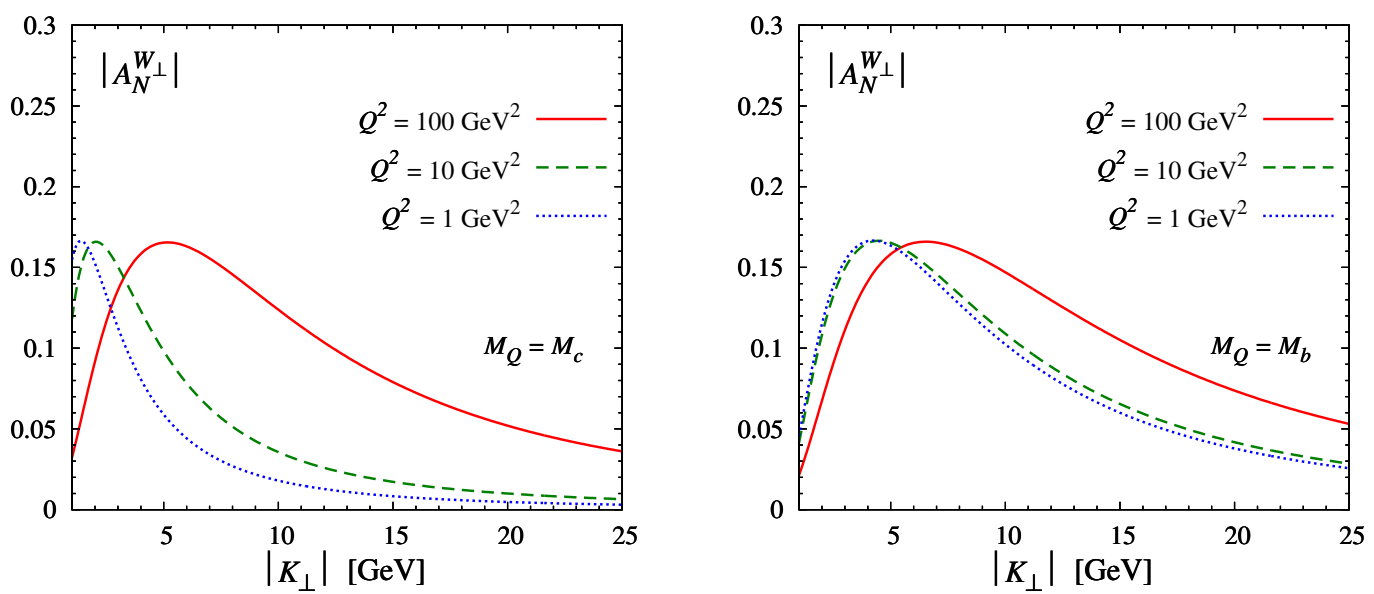

Figure 7. Same as in figure 5, but for the asymmetries $A_{N}^{W_{\perp}}$, with $W_{\perp}=\sin \left(\phi_{S}^{\perp}+\phi_{T}^{\perp}\right)$, $\sin \left(\phi_{S}^{\perp}-3 \phi_{T}^{\perp}\right)$.

\section{Sign change test of the T-odd gluon TMDs}

In this section we discuss the gauge link structure of the gluon TMDs in the process $e p^{\uparrow} \rightarrow$ $e^{\prime} Q \bar{Q} X$ and its consequences. The subprocess $\gamma^{*} g \rightarrow Q \bar{Q}$ probes a gluon correlator with two future pointing Wilson lines, commonly referred to as + links, at small $x$ corresponding to the WW type functions. In contrast, the process $p^{\uparrow} p \rightarrow \gamma$ jet $X$ in the kinematic regime where gluons in the polarized proton dominate, effectively selects the subprocess $q g \rightarrow \gamma q$, which probes a gluon correlator with a + and - link (future and past pointing), at small $x$ corresponding to the dipole type functions. These two processes thus probe distinct and entirely independent T-odd gluon TMDs. In this way processes in $e p$ collisions and $p p$ collisions can be completely complementary. However, it is important to also note that 
one can study processes in $p p$ collisions that are fully related to the ones in $e p$ collisions. For example, the process $p^{\uparrow} p \rightarrow \gamma \gamma X$ in the back-to-back correlation limit [38], probes T-odd gluon TMDs with two past-pointing Wilson lines, which are related by an overall sign change to the ones with two future-pointing links. As a result, we can make the new TMD-formalism prediction that the gluon Sivers function probed in $e p^{\uparrow} \rightarrow e^{\prime} Q \bar{Q} X$ is of opposite sign to the one probed in $p^{\uparrow} p \rightarrow \gamma \gamma X$ :

$$
f_{1 T}^{\perp g\left[e p^{\uparrow} \rightarrow e^{\prime} Q \bar{Q} X\right]}\left(x, p_{T}^{2}\right)=-f_{1 T}^{\perp g\left[p^{\uparrow} p \rightarrow \gamma \gamma X\right]}\left(x, p_{T}^{2}\right),
$$

and the same relation applies to the other two T-odd gluon TMDs, $h_{1}^{g}$ (or $h_{1 T}$ ) and $h_{1 T}^{\perp}$. Here one can consider instead of a $\gamma \gamma$ pair any other color singlet state in $g g$-dominated kinematics, such as $J / \psi \gamma$ or $J / \psi J / \psi$ pairs [39,40]. This is the gluonic analogue of the famous sign change relation between the quark Sivers TMD probed in SIDIS and DrellYan [41]. In our view this provides additional motivation to study gluon Sivers effects at RHIC or AFTER@LHC and compare it to EIC studies in the future, irrespective of any theoretical expectations about WW-type gluon TMDs at (very) small $x$. For T-even functions there is no sign change, such that the process $p p \rightarrow H X[42,43]$ at LHC (or instead of the Higgs boson a (pseudo-)scalar heavy quarkonium state [44]), probes the same $h_{1}^{\perp g}$ function as the process $e p \rightarrow e^{\prime} Q \bar{Q} X$ at EIC:

$$
h_{1}^{\perp g\left[e p \rightarrow e^{\prime} Q \bar{Q} X\right]}\left(x, p_{T}^{2}\right)=h_{1}^{\perp g[p p \rightarrow H X]}\left(x, p_{T}^{2}\right) .
$$

The l.h.s. involves the gluon TMDs with two - links and the r.h.s. with two + links, which for T-even functions are equal. On the other hand, a process like Higgs +jet production probes a more complicated link structure [45]. The same applies to heavy quark-antiquark pair production in proton-proton collisions or proton-nucleus collisions [46], although that process is expected to be TMD factorizing at small $x$ only.

In conclusion, the comparison between TMD observables that can be studied at LHC and at EIC can be related or complementary depending on the process.

\section{Dijet production}

The angular structure of the cross section for the process

$$
e(\ell)+p(P, S) \rightarrow e\left(\ell^{\prime}\right)+\operatorname{jet}\left(K_{1}\right)+\operatorname{jet}\left(K_{2}\right)+X,
$$

is the same as in eqs. (3.2)-(3.4), with

$$
\mathcal{N}=\frac{\alpha^{2} \alpha_{s}}{\pi s \boldsymbol{K}_{\perp}^{2}} \frac{1}{x_{B} y^{2}}
$$

The terms $A_{l}^{U / T}$ at LO receive contributions from two subprocesses, $e q \rightarrow e^{\prime} q g$ and $e g \rightarrow$ $e^{\prime} q \bar{q}$. They can be written in the form

$$
\begin{aligned}
& A_{l}^{U}=\sum_{q, \bar{q}} e_{q}^{2} C_{F} \mathcal{A}_{l}^{e q \rightarrow e q g} f_{1}^{q}\left(x, \boldsymbol{q}_{T}^{2}\right)+\sum_{q} e_{q}^{2} T_{R} \mathcal{A}_{l}^{e g \rightarrow e q \bar{q}} f_{1}^{g}\left(x, \boldsymbol{q}_{T}^{2}\right), \\
& A_{l}^{T}=\sum_{q, \bar{q}} e_{q}^{2} C_{F} \mathcal{A}_{l}^{e q \rightarrow e q g} f_{1 T}^{\perp q}\left(x, \boldsymbol{q}_{T}^{2}\right)+\sum_{q} e_{q}^{2} T_{R} \mathcal{A}_{l}^{e g \rightarrow e q \bar{q}} f_{1 T}^{\perp g}\left(x, \boldsymbol{q}_{T}^{2}\right),
\end{aligned}
$$


where $C_{F}=\left(N_{c}^{2}-1\right) / 2 N_{c}$, with $N_{c}$ being the number of colors. The hard scattering functions $\mathcal{A}_{l}^{e q \rightarrow e q g}$ are the same as the ones calculated in ref. [2] for unpolarized scattering. They read

$$
\begin{aligned}
\mathcal{A}_{0}^{e q \rightarrow e q g} & =\left[1+(1-y)^{2}\right] \mathcal{A}_{U+L}^{\gamma^{*} q \rightarrow q g}-y^{2} \mathcal{A}_{L}^{\gamma^{*} q \rightarrow q g}, \\
\mathcal{A}_{1}^{e q \rightarrow q g} & =(2-y) \sqrt{1-y} \mathcal{A}_{I}^{\gamma^{*} q \rightarrow q g}, \\
\mathcal{A}_{2}^{e q \rightarrow e q g} & =2(1-y) \mathcal{A}_{T}^{\gamma^{*} q \rightarrow q g},
\end{aligned}
$$

where

$$
\begin{aligned}
& \mathcal{A}_{U+L}^{\gamma^{*} q \rightarrow q g}=\frac{1-z}{D_{0}^{2}}\left\{1+z^{2}+\left[2 z(1-z)+4 z^{2}(1-z)^{2}\right] \frac{Q^{2}}{\boldsymbol{K}_{\perp}^{2}}+\left[z^{2}(1-z)^{2}\right]\left[1+(1-z)^{2}\right] \frac{Q^{4}}{\boldsymbol{K}_{\perp}^{4}}\right\} \\
& \mathcal{A}_{L}^{\gamma^{*} q \rightarrow q g}=4 \frac{z^{2}(1-z)^{3}}{D_{0}^{2}} \frac{Q^{2}}{\boldsymbol{K}_{\perp}^{2}}, \\
& \mathcal{A}_{I}^{\gamma^{*} q \rightarrow q g}=-4 \frac{z^{2}(1-z)^{2}}{D_{0}^{2}}\left[1+(1-z)^{2} \frac{Q^{2}}{\boldsymbol{K}_{\perp}^{2}}\right] \frac{Q}{\left|\boldsymbol{K}_{\perp}\right|}, \\
& \mathcal{A}_{T}^{\gamma^{*} q \rightarrow q g}=2 \frac{z^{2}(1-z)^{3}}{D_{0}^{2}} \frac{Q^{2}}{\boldsymbol{K}_{\perp}^{2}}
\end{aligned}
$$

with

$$
D_{0} \equiv D_{0}\left(z, \frac{Q^{2}}{\boldsymbol{K}_{\perp}^{2}}\right)=1+z(1-z) \frac{Q^{2}}{\boldsymbol{K}_{\perp}^{2}} .
$$

Only the process $e g \rightarrow e^{\prime} q \bar{q}$ contributes to the terms $B_{m}^{U}, B_{m}^{T}$ and $B_{m}^{\prime T}$, which can be written as

$$
\begin{aligned}
B_{m}^{U} & =\sum_{q} e_{q}^{2} T_{R} \mathcal{B}_{m}^{e g \rightarrow e q \bar{q}} \frac{\boldsymbol{q}_{T}^{2}}{M_{p}^{2}} h_{1}^{\perp g}\left(x, \boldsymbol{q}_{T}^{2}\right), \quad B_{m}^{T}=B_{m}^{U} \frac{\left|\boldsymbol{q}_{T}\right|}{M_{p}} \frac{h_{1 T}^{\perp g}\left(x, \boldsymbol{q}_{T}^{2}\right)}{h_{1}^{\perp g}\left(x, \boldsymbol{q}_{T}^{2}\right)}, \\
B_{m}^{\prime T} & =B_{m}^{U} \frac{M_{p}}{\left|\boldsymbol{q}_{T}\right|} \frac{h_{1 T}^{g}\left(x, \boldsymbol{q}_{T}^{2}\right)}{h_{1}^{\perp g}\left(x, \boldsymbol{q}_{T}^{2}\right)} .
\end{aligned}
$$

The explicit expressions for $\mathcal{A}_{l}^{e g \rightarrow e q \bar{q}}$ and $\mathcal{B}_{m}^{e g \rightarrow e q \bar{q}}$ can be obtained from the corresponding ones for the process $e g \rightarrow e Q \bar{Q}$, presented in the previous section, by taking the limit $M_{Q} \rightarrow 0$.

For the process under study it is possible to define azimuthal moments similar to those introduced in the previous section for heavy quark pair production. The denominators of the various asymmetries for dijet production will be given by

$$
A_{0}^{U}=\sum_{q, \bar{q}} e_{q}^{2} C_{F} \mathcal{A}_{0}^{e q \rightarrow e q g} f_{1}^{q}\left(x, \boldsymbol{q}_{T}^{2}\right)+\sum_{q} e_{q}^{2} T_{R} \mathcal{A}_{0}^{e g \rightarrow e q \bar{q}} f_{1}^{g}\left(x, \boldsymbol{q}_{T}^{2}\right) .
$$

In a kinematic region where $x$ is small enough, such that the quark contributions can be neglected, the maximal values of the asymmetries for dijet production will be the same as the corresponding ones for heavy quark pair production in the limit $M_{Q} \rightarrow 0$. We then recover the expressions of $[8,18]$ for the linearly polarized gluon asymmetries. In [47] the 

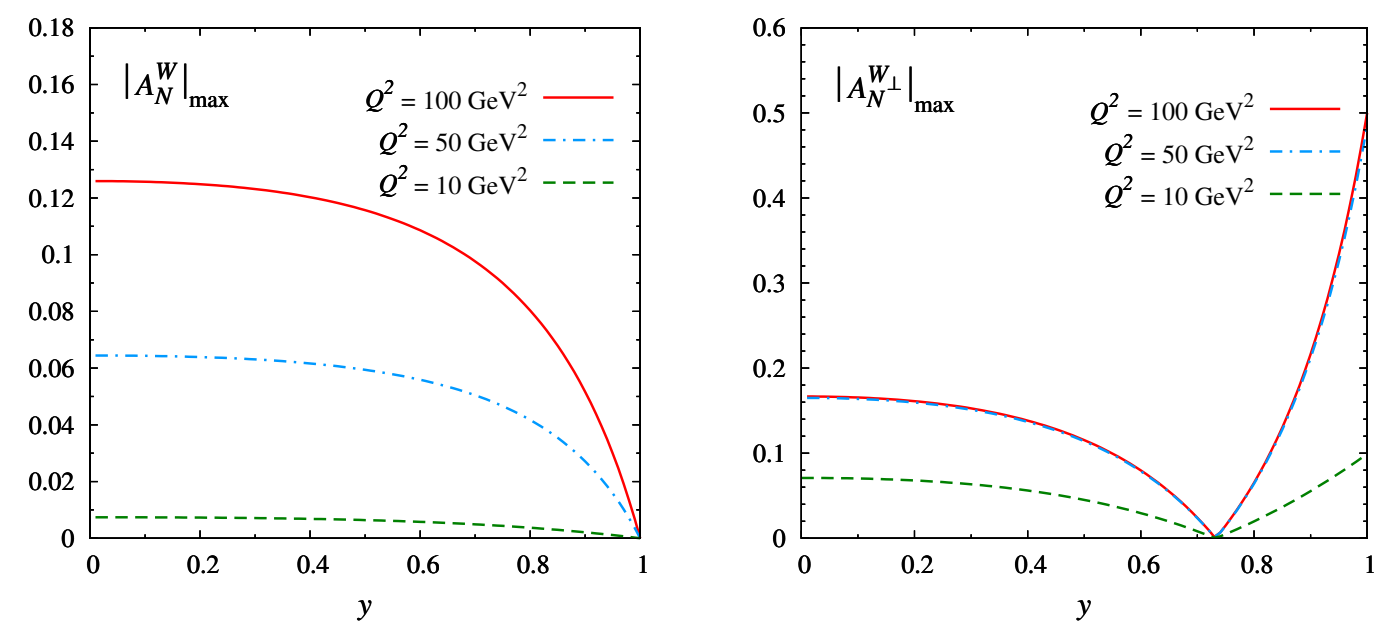

Figure 8. Upper bounds $\left|A_{N}^{W}\right|_{\max }$, with $W=\sin \left(\phi_{S}+\phi_{T}\right), \sin \left(\phi_{S}-3 \phi_{T}\right)$ (left panel), and $\left|A_{N}^{W^{\perp}}\right|_{\max }$, with $W_{\perp}=\sin \left(\phi_{S}^{\perp}+\phi_{T}^{\perp}\right), \sin \left(\phi_{S}^{\perp}-3 \phi_{T}^{\perp}\right)$ (right panel), for the process $e p \rightarrow e^{\prime}$ jet jet X. Estimates are given as a function of $y$, at different values of $K_{\perp} \geq 4 \mathrm{GeV}$ (chosen in such a way that the asymmetries are maximal) and $Q^{2}$, with $z=0.5$.

effects of the WW $h_{1}^{\perp g}$ in a CGC-formalism calculation including BK evolution with and without running coupling effects were studied for dijet production at EIC. The results for $v_{2} \equiv\left\langle\cos 2\left(\phi_{T}-\phi_{\perp}\right)\right\rangle$ were found to be large for $\sqrt{s}=100 \mathrm{GeV}, y=1, z=0.5$ and $Q^{2}=4 K_{\perp}^{2}$, reaching values up to 0.4 at larger transverse momentum of the dijet pair, close to the bound of 0.5. Although the selected kinematics do not correspond to small $x$ values unless $K_{\perp}$ is rather small for dijet production considerations $\left(K_{\perp}<5 \mathrm{GeV}\right.$ in order for $x_{B}<0.01$ ), the theoretically predicted logarithmic suppression [18] for $p_{T} \ll Q_{s}$ may thus only become apparent for much smaller $x$ values when $Q_{s}$ is much larger than typically considered for EIC.

Our MV model estimates for $\left|\left\langle\cos 2\left(\phi_{T}-\phi_{\perp}\right)\right\rangle\right|$ and $\left|\left\langle\cos 2 \phi_{T}\right\rangle\right|$ in the dijet case are very similar to the charm production case for $Q^{2} \geq 10 \mathrm{GeV}^{2}$. For smaller $Q^{2}$, which corresponds to smaller $x$ values, the asymmetries are unfortunately significantly smaller than the $Q^{2}=1 \mathrm{GeV}^{2}$ curves for $M_{Q}=M_{c}$. For this reason we do not include separate plots for those asymmetries. Note that upon inclusion of the quark contributions to the denominator, the asymmetries will decrease further in magnitude.

For the T-odd gluon TMD asymmetries, upper bounds (without quark contributions) are shown in figures 8 and 9. Again they are similar to the $M_{Q}=M_{c}$ case for large $Q^{2}$ and substantially smaller for small $Q^{2}$. We emphasize that at small $x$ these bounds are not expected to be satisfied. The simple models presented could be used to scale the asymmetries to more reasonable values in the small- $x$ case. We have selected $K_{\perp} \geq 4 \mathrm{GeV}$ based on the multijet analysis of small $x_{B}$ HERA data in [48].

\section{Conclusions}

We have studied the azimuthal asymmetries in heavy quark pair and dijet production in DIS process, which provide direct access to the WW-type gluon TMDs. We found that in these 

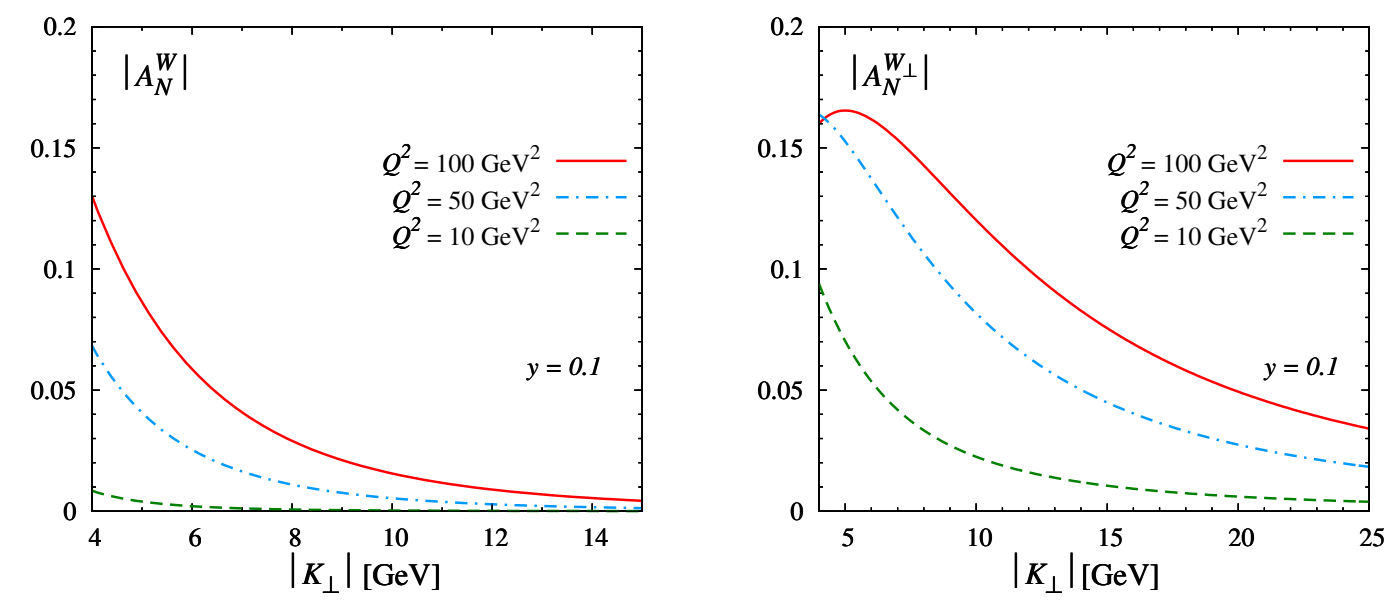

Figure 9. Estimates of $\left|A_{N}^{W}\right|$, with $W=\sin \left(\phi_{S}+\phi_{T}\right), \sin \left(\phi_{S}-3 \phi_{T}\right)$ (left panel), and $\left|A_{N}^{W_{\perp}}\right|$, with $W_{\perp}=\sin \left(\phi_{S}^{\perp}+\phi_{T}^{\perp}\right), \sin \left(\phi_{S}^{\perp}-3 \phi_{T}^{\perp}\right)$ (right panel), for the process $e p \rightarrow e^{\prime}$ jet jet X. The asymmetries are calculated at $z=0.5, y=0.1$ and at different values of $Q^{2}$, as a function of $\left|\boldsymbol{K}_{\perp}\right|(\geq 4 \mathrm{GeV})$.

processes the gluon TMDs arise in the same way as the quark TMDs do in semi-inclusive DIS. The measurements of these azimuthal asymmetries at an EIC would allow a sign change test of the T-odd gluon TMDs by comparing to corresponding observables at RHIC and AFTER@LHC. Furthermore, it was shown that the asymmetries maximally allowed by the positivity bounds are rather sizeable, except in some kinematic limits. Although WWtype gluon TMDs suffer from suppression in the small- $x$ region, the effects from linearly polarized gluons are still expected to be sizeable in heavy quark pair production at an EIC, but less so in dijet production because of the requirement that the transverse momentum of each jet must be large. For the Sivers gluon TMD the bounds are always maximal and provide little guidance, especially in the small- $x$ region where the WW-type function is suppressed by a factor of $x$ with respect to the unpolarized gluon TMD. For the other two T-odd gluon TMDs that arise with $\sin \left(\phi_{S}+\phi_{T}\right)$ and $\sin \left(\phi_{S}-3 \phi_{T}\right)$ modulations, their ratio can be exploited to test small- $x$ expectations. We have provided some simple model expectations for that ratio. It will also be interesting to compare these quantities to the dipole type T-odd gluon TMDs that arise in other processes, such as virtual photon plus jet production in polarized proton-proton collisions at RHIC. This allows to experimentally test the theoretical expectation that the WW and dipole type T-odd gluon TMDs have different small $x$ asymptotic behavior. Hence, the studies of these asymmetries could form a prominent part of both the spin physics program and the small- $x$ physics program at a future EIC.

\section{Acknowledgments}

This research is partially supported by the European Research Council (ERC) under the FP7 "Ideas" programme (grant agreement No. 320389, QWORK) and the European Union's Horizon 2020 research and innovation programme (grant agreement No. 647981, 3DSPIN). 
Open Access. This article is distributed under the terms of the Creative Commons Attribution License (CC-BY 4.0), which permits any use, distribution and reproduction in any medium, provided the original author(s) and source are credited.

\section{References}

[1] D. Boer, S.J. Brodsky, P.J. Mulders and C. Pisano, Direct Probes of Linearly Polarized Gluons inside Unpolarized Hadrons, Phys. Rev. Lett. 106 (2011) 132001 [arXiv:1011.4225] [INSPIRE].

[2] C. Pisano, D. Boer, S.J. Brodsky, M.G.A. Buffing and P.J. Mulders, Linear polarization of gluons and photons in unpolarized collider experiments, JHEP 10 (2013) 024 [arXiv:1307.3417] [INSPIRE].

[3] D. Boer et al., Gluons and the quark sea at high energies: Distributions, polarization, tomography, arXiv:1108.1713 [INSPIRE].

[4] A. Accardi et al., Electron Ion Collider: The Next QCD Frontier - Understanding the glue that binds us all, arXiv:1212.1701 [INSPIRE].

[5] D.W. Sivers, Single Spin Production Asymmetries from the Hard Scattering of Point-Like Constituents, Phys. Rev. D 41 (1990) 83 [INSPIRE].

[6] S.J. Brodsky, F. Fleuret, C. Hadjidakis and J.P. Lansberg, Physics Opportunities of a Fixed-Target Experiment using the LHC Beams, Phys. Rept. 522 (2013) 239 [arXiv: 1202.6585] [INSPIRE].

[7] J.P. Lansberg et al., Spin physics and TMD studies at A Fixed-Target ExpeRiment at the LHC (AFTER@LHC), EPJ Web Conf. 85 (2015) 02038 [arXiv:1410.1962] [INSPIRE].

[8] F. Dominguez, C. Marquet, B.-W. Xiao and F. Yuan, Universality of Unintegrated Gluon Distributions at small x, Phys. Rev. D 83 (2011) 105005 [arXiv:1101.0715] [INSPIRE].

[9] F. Dominguez, B.-W. Xiao and F. Yuan, $k_{t}$-factorization for Hard Processes in Nuclei, Phys. Rev. Lett. 106 (2011) 022301 [arXiv: 1009. 2141] [INSPIRE].

[10] D. Boer, M.G. Echevarria, P.J. Mulders and J. Zhou, Single spin asymmetries from a single Wilson loop, Phys. Rev. Lett. 116 (2016) 122001 [arXiv:1511.03485] [INSPIRE].

[11] A. Schäfer and J. Zhou, Transverse single spin asymmetry in hadronic $\eta c, b$ production, Phys. Rev. D 88 (2013) 014008 [arXiv: 1302.4600] [INSPIRE].

[12] K. Kanazawa, Y. Koike, A. Metz and D. Pitonyak, Transverse single-spin asymmetries in proton-proton collisions at the AFTER@LHC experiment, Adv. High Energy Phys. 2015 (2015) 257934 [arXiv: 1502.04021] [INSPIRE].

[13] J.P. Ma and C. Wang, QCD factorization for quarkonium production in hadron collisions at low transverse momentum, Phys. Rev. D 93 (2016) 014025 [arXiv: 1509.04421] [InSPIRE].

[14] D. Boer, C. Lorcé, C. Pisano and J. Zhou, The gluon Sivers distribution: status and future prospects, Adv. High Energy Phys. 2015 (2015) 371396 [arXiv:1504.04332] [INSPIRE].

[15] P.J. Mulders and J. Rodrigues, Transverse momentum dependence in gluon distribution and fragmentation functions, Phys. Rev. D 63 (2001) 094021 [hep-ph/0009343] [INSPIRE].

[16] S. Meissner, A. Metz and K. Goeke, Relations between generalized and transverse momentum dependent parton distributions, Phys. Rev. D 76 (2007) 034002 [hep-ph/0703176] [INSPIRE]. 
[17] L.D. McLerran and R. Venugopalan, Computing quark and gluon distribution functions for very large nuclei, Phys. Rev. D 49 (1994) 2233 [hep-ph/9309289] [INSPIRE].

[18] A. Metz and J. Zhou, Distribution of linearly polarized gluons inside a large nucleus, Phys. Rev. D 84 (2011) 051503 [arXiv: 1105.1991] [INSPIRE].

[19] F. Dominguez, J.-W. Qiu, B.-W. Xiao and F. Yuan, On the linearly polarized gluon distributions in the color dipole model, Phys. Rev. D 85 (2012) 045003 [arXiv:1109.6293] [INSPIRE].

[20] J.L. Albacete, N. Armesto, J.G. Milhano, P. Quiroga-Arias and C.A. Salgado, AAMQS: A non-linear QCD analysis of new HERA data at small-x including heavy quarks, Eur. Phys. J. C 71 (2011) 1705 [arXiv: 1012.4408] [INSPIRE].

[21] T. Lappi and H. Mäntysaari, Single inclusive particle production at high energy from HERA data to proton-nucleus collisions, Phys. Rev. D 88 (2013) 114020 [arXiv:1309.6963] [INSPIRE].

[22] J.L. Albacete, N. Armesto, A. Kovner, C.A. Salgado and U.A. Wiedemann, Energy dependence of the Cronin effect from nonlinear QCD evolution, Phys. Rev. Lett. 92 (2004) 082001 [hep-ph/0307179] [INSPIRE].

[23] R. Enberg, K.J. Golec-Biernat and S. Munier, The High energy asymptotics of scattering processes in QCD, Phys. Rev. D 72 (2005) 074021 [hep-ph/0505101] [INSPIRE].

[24] K.J. Golec-Biernat and M. Wüsthoff, Saturation effects in deep inelastic scattering at low $Q^{2}$ and its implications on diffraction, Phys. Rev. D 59 (1998) 014017 [hep-ph/9807513] [INSPIRE].

[25] X. Ji, J.-W. Qiu, W. Vogelsang and F. Yuan, A Unified picture for single transverse-spin asymmetries in hard processes, Phys. Rev. Lett. 97 (2006) 082002 [hep-ph/0602239] [INSPIRE].

[26] J. Zhou, F. Yuan and Z.-T. Liang, Hyperon Polarization in Unpolarized Scattering Processes, Phys. Rev. D 78 (2008) 114008 [arXiv:0808.3629] [InSPIRE].

[27] J.-w. Qiu and G.F. Sterman, Single transverse spin asymmetries, Phys. Rev. Lett. 67 (1991) 2264 [INSPIRE].

[28] X.-D. Ji, Gluon correlations in the transversely polarized nucleon, Phys. Lett. B 289 (1992) 137 [INSPIRE].

[29] H. Beppu, Y. Koike, K. Tanaka and S. Yoshida, Contribution of Twist-3 Multi-Gluon Correlation Functions to Single Spin Asymmetry in Semi-Inclusive Deep Inelastic Scattering, Phys. Rev. D 82 (2010) 054005 [arXiv: 1007.2034] [INSPIRE].

[30] A. Schäfer and J. Zhou, A note on the scale evolution of tri-gluon correlations, arXiv: 1308.4961 [INSPIRE].

[31] M. Burkardt, Quark correlations and single spin asymmetries, Phys. Rev. D 69 (2004) 057501 [hep-ph/0311013] [INSPIRE].

[32] C. Lorcé, The light-front gauge-invariant energy-momentum tensor, JHEP 08 (2015) 045 [arXiv: 1502.06656] [INSPIRE].

[33] J. Zhou, Note on the scale dependence of the Burkardt sum rule, Phys. Rev. D 92 (2015) 074016 [arXiv: 1507.02819] [INSPIRE]. 
[34] J. Zhou, Transverse single spin asymmetries at small $x$ and the anomalous magnetic moment, Phys. Rev. D 89 (2014) 074050 [arXiv: 1308.5912] [INSPIRE].

[35] J. Bartels, B.I. Ermolaev and M.G. Ryskin, Nonsinglet contributions to the structure function g1 at small $x, Z$. Phys. C 70 (1996) 273 [hep-ph/9507271] [InSPIRE].

[36] T. Brodkorb and E. Mirkes, Complete $O\left(\alpha-S^{2}\right)$ in deep inelastic scattering, Z. Phys. C 66 (1995) 141 [hep-ph/9402362] [INSPIRE].

[37] D. Boer and P.J. Mulders, Time reversal odd distribution functions in leptoproduction, Phys. Rev. D 57 (1998) 5780 [hep-ph/9711485] [INSPIRE].

[38] J.-W. Qiu, M. Schlegel and W. Vogelsang, Probing Gluonic Spin-Orbit Correlations in Photon Pair Production, Phys. Rev. Lett. 107 (2011) 062001 [arXiv:1103.3861] [InSPIRE].

[39] W.J. den Dunnen, J.P. Lansberg, C. Pisano and M. Schlegel, Accessing the Transverse Dynamics and Polarization of Gluons inside the Proton at the LHC, Phys. Rev. Lett. 112 (2014) 212001 [arXiv:1401.7611] [INSPIRE].

[40] J.-P. Lansberg and H.-S. Shao, Double-quarkonium production at a fixed-target experiment at the LHC (AFTER@LHC), Nucl. Phys. B 900 (2015) 273 [arXiv: 1504.06531] [INSPIRE].

[41] J.C. Collins, Leading twist single transverse-spin asymmetries: Drell-Yan and deep inelastic scattering, Phys. Lett. B 536 (2002) 43 [hep-ph/0204004] [INSPIRE].

[42] P. Sun, B.-W. Xiao and F. Yuan, Gluon Distribution Functions and Higgs Boson Production at Moderate Transverse Momentum, Phys. Rev. D 84 (2011) 094005 [arXiv:1109.1354] [INSPIRE].

[43] D. Boer, W.J. den Dunnen, C. Pisano, M. Schlegel and W. Vogelsang, Linearly Polarized Gluons and the Higgs Transverse Momentum Distribution, Phys. Rev. Lett. 108 (2012) 032002 [arXiv: 1109.1444] [INSPIRE].

[44] D. Boer and C. Pisano, Polarized gluon studies with charmonium and bottomonium at LHCb and AFTER, Phys. Rev. D 86 (2012) 094007 [arXiv: 1208.3642] [InSPIRE].

[45] D. Boer and C. Pisano, Impact of gluon polarization on Higgs boson plus jet production at the LHC, Phys. Rev. D 91 (2015) 074024 [arXiv:1412.5556] [INSPIRE].

[46] E. Akcakaya, A. Schäfer and J. Zhou, Azimuthal asymmetries for quark pair production in pA collisions, Phys. Rev. D 87 (2013) 054010 [arXiv: 1208.4965] [INSPIRE].

[47] A. Dumitru, T. Lappi and V. Skokov, Distribution of Linearly Polarized Gluons and Elliptic Azimuthal Anisotropy in Deep Inelastic Scattering Dijet Production at High Energy, Phys. Rev. Lett. 115 (2015) 252301 [arXiv: 1508.04438] [INSPIRE].

[48] ZEUS collaboration, S. Chekanov et al., Multijet production at low x(Bj) in deep inelastic scattering at HERA, Nucl. Phys. B 786 (2007) 152 [arXiv:0705.1931] [INSPIRE]. 